University of Nebraska - Lincoln

DigitalCommons@University of Nebraska - Lincoln

Uniformed Services University of the Health

Sciences

U.S. Department of Defense

2011

\title{
A systematic review of experimental infections with enterotoxigenic Escherichia coli (ETEC)
}

\author{
Chad K. Porter \\ Naval Medical Research Center, chad.porter@med.navy.mil \\ Mark S. Riddle \\ Naval Medical Research Center \\ David R. Tribble \\ Uniformed Services University of the Health Sciences
}

A. Louis Bougeois

Johns Hopkins University

Robin McKenzie

Johns Hopkins University

See next page for additional authors

Follow this and additional works at: https://digitalcommons.unl.edu/usuhs

Part of the Medicine and Health Sciences Commons

Porter, Chad K.; Riddle, Mark S.; Tribble, David R.; Bougeois, A. Louis; McKenzie, Robin; Isidean, Sandra D.; Sebeny, Peter; and Savarino, Stephen J., "A systematic review of experimental infections with enterotoxigenic Escherichia coli (ETEC)" (2011). Uniformed Services University of the Health Sciences. 94.

https://digitalcommons.unl.edu/usuhs/94

This Article is brought to you for free and open access by the U.S. Department of Defense at DigitalCommons@University of Nebraska - Lincoln. It has been accepted for inclusion in Uniformed Services University of the Health Sciences by an authorized administrator of DigitalCommons@University of Nebraska Lincoln. 


\section{Authors}

Chad K. Porter, Mark S. Riddle, David R. Tribble, A. Louis Bougeois, Robin McKenzie, Sandra D. Isidean, Peter Sebeny, and Stephen J. Savarino 
Review

\title{
A systematic review of experimental infections with enterotoxigenic Escherichia coli (ETEC) ${ }^{2 / 2}$
}

\author{
Chad K. Porter ${ }^{a}, *$, Mark S. Riddle ${ }^{a}$, David R. Tribble ${ }^{b}$, A. Louis Bougeois ${ }^{c}$, Robin McKenzie $^{c}$, \\ Sandra D. Isidean ${ }^{\mathrm{a}}$, Peter Sebeny ${ }^{\mathrm{d}}$, Stephen J. Savarino ${ }^{\mathrm{a}}$ \\ a Naval Medical Research Center, Enteric Diseases Department, Silver Spring, MD, United States \\ ${ }^{\mathrm{b}}$ Uniformed Services University of the Health Sciences, Bethesda, MD, United States \\ c Johns Hopkins University, Baltimore, MD, United States \\ d Naval Medical Research Unit No. 3, Cairo, Egypt
}

\section{A R T I C L E I N F O}

\section{Article history:}

Received 10 February 2011

Received in revised form 4 May 2011

Accepted 9 May 2011

Available online 25 May 2011

\section{Keywords:}

Enterotoxigenic Escherichia coli

Challenge

Clinical trial

\begin{abstract}
A B S T R A C T
Volunteer challenge with enterotoxigenic Escherichia coli (ETEC) has been used for four decades to elucidate the pathogenesis and immune responses and assess efficacy of various interventions. We performed a systematic review of these studies and a meta-analysis of individual patient-level data (IPD) from a subset of studies using standard methodology.

We identified 27 studies of 11 ETEC strains administered to 443 naive subjects at doses from $1 \times 10^{6}$ to $1 \times 10^{10}$ colony forming units (cfu). Diarrhea attack rates varied by strain, dose and enterotoxin. Similar rates were seen at doses of $5 \times 10^{8}$ to $1 \times 10^{10} \mathrm{cfu}$ with the three most commonly used strains B7A, E24377A, H10407. In IPD analysis, the highest diarrhea attack rates were seen with strains B7A, H10407 and E24377A. The H10407 induced significantly higher stool output than the other strains. Additionally, the rate of output was different across strains.

The risk of diarrhea, abdominal cramps, nausea and headaches differed significantly by ETEC strain. An increased risk of nausea, abdominal cramps and headaches was seen for females. Baseline anti-LT IgG titers appeared to be associated with a decrease risk of diarrhea outcomes, a trend not seen with anti-LT IgA or seen consistently with anti-colonization factor antibodies. Neither early antibiotic treatment nor diarrhea duration significantly affected the frequency or magnitude of serologic responses.

These studies have served as an invaluable tool in understanding disease course, pathogenicity, innate immune responses and an early assessment of product efficacy. When designing and planning experimental ETEC infection studies in this age of increased ethical scrutiny and growing appreciation of post-infectious sequelae, better understanding of available data is essential.
\end{abstract}

Published by Elsevier Ltd.

\section{Contents}

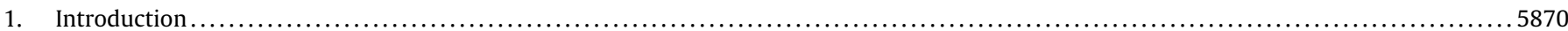

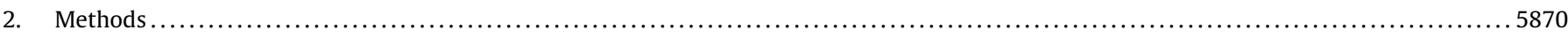

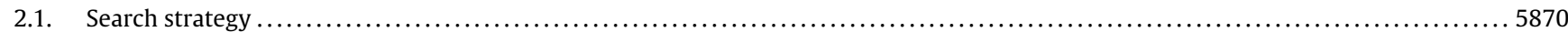

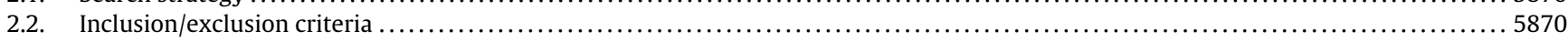

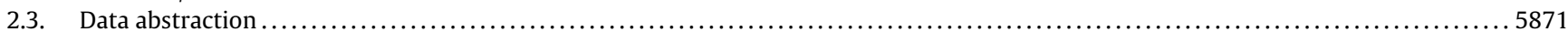

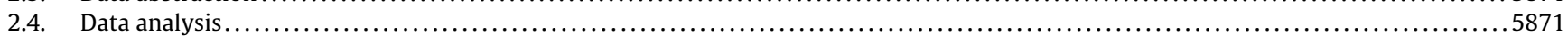

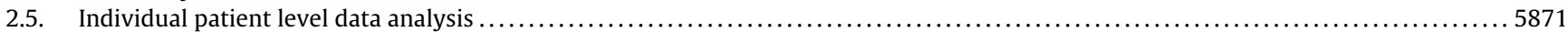

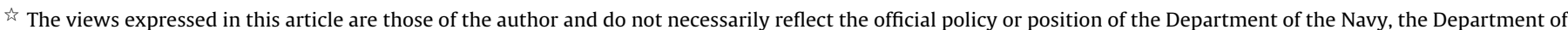
Defense, nor the US Government. The study protocol was approved by the Naval Medical Research Center Institutional Review Board in compliance with all applicable Federal regulations governing the protection of human subjects.

* Corresponding author. Tel.: +1 3013197505.

E-mail address: chad.porter@med.navy.mil (C.K. Porter).
} 


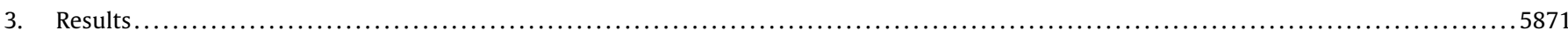

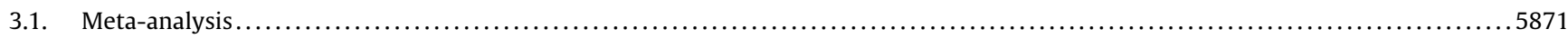

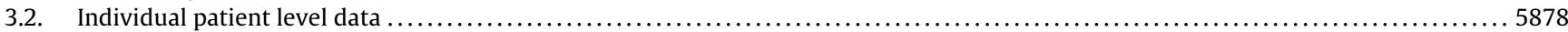

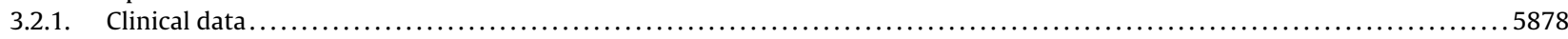

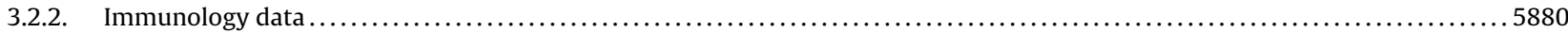

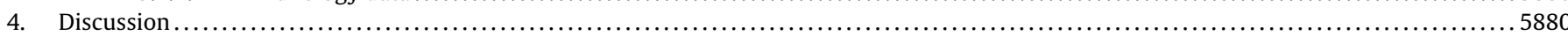

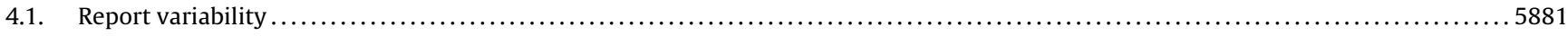

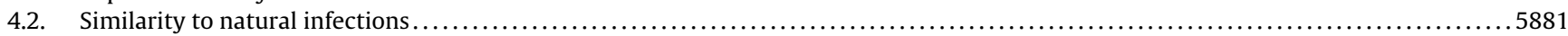

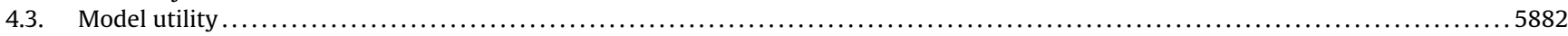

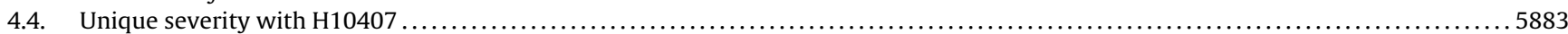

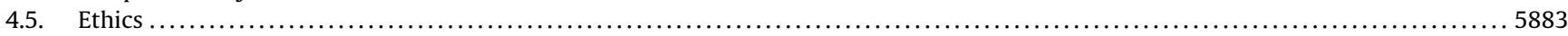

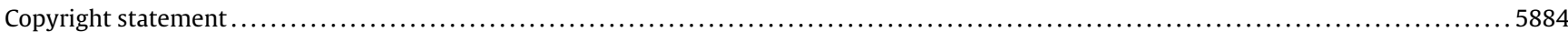

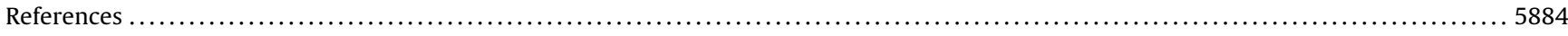

\section{Introduction}

Over the past 40 years, the enterotoxigenic E. coli (ETEC) human challenge model has been used to elucidate the pathogenesis and immune responses associated with ETEC infection as well as to test the efficacy of investigational drugs and vaccines. The initial experimental infection, published in 1971, was a landmark study establishing ETEC as the organism responsible for causing acute, cholera-like illness in a U.S. soldier in Vietnam [1]. In this classic paper, researchers demonstrated that while porcine and human isolates of disease-causing $E$. coli were both capable of inducing fluid excretion in rabbit ileal loops, only human isolates were capable of causing disease in human subjects. It was later discovered that the difference in the two strains was the species-specific tropism of the intestinal colonization factor fimbriae.

Since this Landmark publication, ETEC has been established as the most common cause of diarrhea in travelers as well as in young children in resource-limited regions of the world. As such, it has also become the focus of vaccine development efforts [2,3]. Since an immune correlate of protection has yet to be established and physiologically relevant animal models are lacking, researchers have frequently relied on the use of vaccine-challenge studies in the early clinical development of investigational products. This mechanism has been supportive for vaccine development efforts for cholera, an enteric pathogen with similar disease mechanisms [4] as well as other non-enteric pathogens [5]. The basic concept of the ETEC challenge study is to select a well-characterized, antibiotic susceptible organism that has been associated with diarrhea and related gastrointestinal symptoms. Under close inpatient supervision, the strain is fed to volunteers at a dose that induces diarrhea. Illness is often curtailed by early antibiotic treatment. Preliminary protective efficacy is then calculated by comparing the diarrhea attack rates in subjects receiving an investigational product with subjects receiving placebo.

Here, we have performed a systematic review to thoroughly examine the published literature and other unpublished data and compiled aggregate information regarding the pathogenicity, virulence, and immune responses observed in experimental ETEC infections. Our purpose was to understand the relationship between clinical manifestations of infection and ETEC virulence factors as well as identify potentially important hostspecific factors similarly associated with clinical outcomes. The expected outcome is a better understanding of experimental ETEC infections with regards to factors inherent to the CFtoxin profiles of the ETEC strains tested, and factors external to the organism that may affect pathogenicity such as inoculum preparation and administration procedures, variability in study populations. Our findings can be applied to the design and interpretation of future studies with previously untested ETEC strains.

\section{Methods}

This study was a systematic review of the published and unpublished literature to evaluate specific outcomes in subjects participating in experimental ETEC infection studies using the accepted principles of good methodological design [6,7]. The methodology included the formulation of an analytic framework with the development of key questions to be answered by systematic reviews of the scientific literature. For each question, the systematic review included eligibility criteria for available evidence, standardized data abstraction, critical appraisal of the quality of the evidence, analysis of the data (including a determination of the appropriateness of applying meta-analysis), and interpretation of the results.

In addition to study-level information, a subset of studies with known similarities in specific outcomes was identified for the compilation and analysis of individual patient data. In addition to evaluating factors inconsistently reported in the published literature, analysis of pooled individual patient-level data (IPD) affords the opportunity for more detailed analyses while avoiding some of the potential biases inherent in analyzing summary statistics of study participants [8].

\subsection{Search strategy}

A comprehensive retrieval of information was conducted by initially performing searches of electronic bibliographies including MEDLINE, EMBASE, CINAHL, and the Cochrane Library. All searches were limited to human studies and started with the term ETEC which was then followed by the addition of the following terms: infection, efficacy, experimental, inpatient and challenge. In addition, MEDLINE searches were conducted using major medical subject headings (MeSH) determined from articles known to be eligible. Additionally, a manual search of the bibliographies of retrieved articles was performed. Conference proceedings, book chapters and technical reports were also reviewed to identify potential studies. Because this study was not limited to published articles, we consulted with experts in the field of ETEC research to identify any previously unidentified eligible studies. Studies had to be completed and/or otherwise available prior to January 2009 to be included in this analysis. All articles, publications and abstracts were reviewed to determine if they met the eligibility criteria, assessed by two independent reviewers (CP and PS).

\subsection{Inclusion/exclusion criteria}

This study was limited to experimental infection studies in which subjects received live, unattenuated strains of ETEC bacteria either as part of the development of an experimental challenge model, for characterization of strain pathogenicity and/or 
immunogenicity or as controls for the evaluation of a vaccine, prophylaxis or treatment product. Subjects receiving an investigational product prior to, or after ingesting the ETEC inoculum were not included in the analysis. The search was limited to studies reported in the English language.

\subsection{Data abstraction}

Two reviewers (CP and SI) extracted the data using a pre-tested data extraction form. Bibliographic information, study design description, study years, geographic location, population characteristics, primary outcome measures, inoculum and strain information and other study characteristics necessary to assess the key parameters and to evaluate heterogeneity were included. For studies involving a vaccine or treatment arm, only data from the placebo control arm were extracted. Abstraction was not blinded to any study characteristic such as author, journal or year of publication.

Data were entered separately by each of the reviewers into a Microsoft Access database. Discrepancies in data points were evaluated by a third party and resolved by consensus. Results were tabulated from individual studies.

\subsection{Data analysis}

Heterogeneity was assessed using a $\chi^{2}$ heterogeneity statistic, and potential sources of heterogeneity were assessed graphically by Forest plots and non-parametric methods (e.g., Kruskal-Wallis, Mann-Whitney $U$-test) to compare differences in incidence between two or more groups of a given study characteristic. In the case of parameters where only a few studies were found, a median and range of estimates were reported. For summary purposes, point estimates and standard 95\% confidence intervals were combined using a random-effects model with methodology developed by DerSimonian and Laird [9]. As the principle purpose of this systematic review was to summarize studies reporting diarrhea incidence following experimental infection, publication bias was not assessed; as such, the concern for non-published findings due to negative studies or disappointing results was considered minimal.

The independent study characteristics that were evaluated included strain and quantity of ETEC administered, inoculum administration procedures and volunteer characteristics. These were assessed in relation to their effect on multiple outcomes such as diarrheal attack rates, disease severity, incubation periods, nondiarrheal symptoms and qualitative immune responses to both the colonization factor and the toxin (when appropriate).

\subsection{Individual patient level data analysis}

For the IPD analysis, only studies for which IPD was obtained were included. Also, these analyses were limited to studies utilizing the same inoculum administration procedures and outcome definitions (diarrhea, immune response, etc.). This analysis included 7 separate studies evaluating 12 strain/dose combinations and a total of 134 subjects. All but one of the studies (evaluating two doses of two separate ETEC strains) were performed by the same principal investigator (PI) at the same clinical facility. For that study, we evaluated the impact of the different clinical site and PI on the clinical outcomes. The lack of significant differences in study populations, or clinical or immunologic outcomes for the lone strain/dose combination in question, led us to combine the data from the multiple studies as if they were performed as a single clinical trial instead of utilizing multilevel and/or hierarchical models to allow for adjustment of between-trial variance (deemed of minimal impact for this unique dataset) $[10,11]$. Outcomes evaluated were the same as those for the over-arching meta-analysis. Post hoc

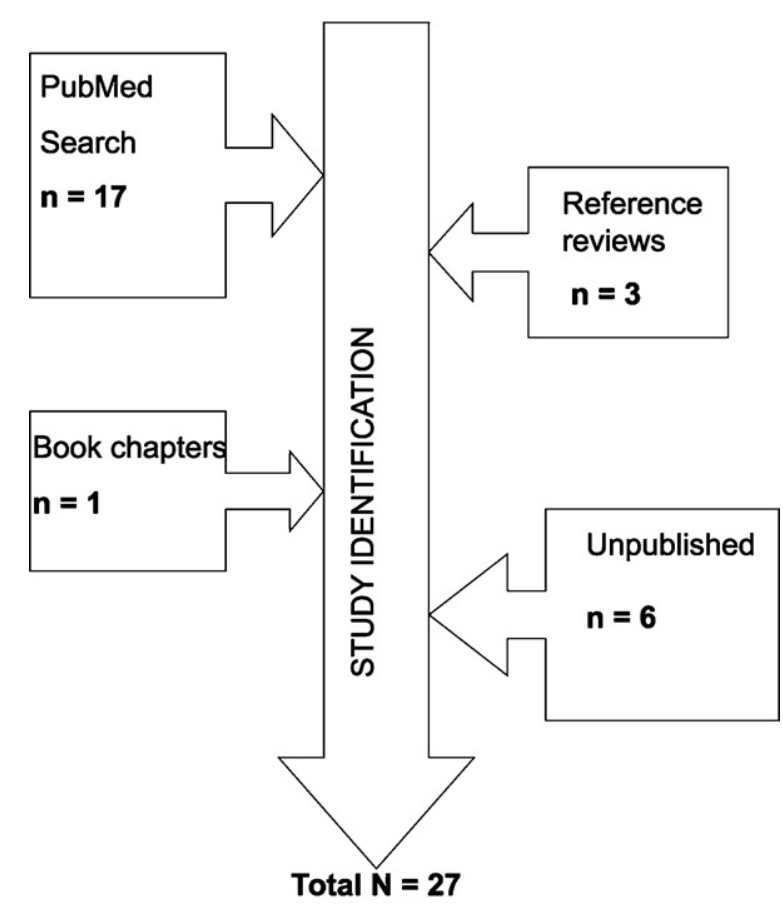

Fig. 1. Flow diagram for studies included in systematic review.

analyses of non-parametric continuous variables were performed using a bonferroni-adjusted alpha for pair-wise comparisons.

\section{Results}

\subsection{Meta-analysis}

A total of 27 studies were identified for inclusion (Fig. 1). A thorough review of the published literature identified a total of 22 reports of experimental ETEC infection. However, 4 publications reported different aspects of only 2 different clinical trials [12-15], yielding 19 individual studies. One additional study was identified in two separate book chapters with topics related to ETEC vaccine pathogenicity and vaccine development [16,17]. We also included 6 unpublished studies for which three principal investigators have been extensively involved. These included administration of 7 different ETEC strains (E24377A, H10407, LSN03-016011/A, WS0115A, DS26-1) in experimental infection or preliminary protective efficacy studies at the inpatient facilities at Johns Hopkins University. We excluded one study that did not report any data on clinical outcomes following ETEC ingestion [18]. A complete listing of all included studies is shown in Table 1 .

The majority (70\%) of the published studies were printed in a 12-year span between 1977 and 1988. However, the past decade has seen an increase in the number of experimental ETEC infection studies, many of which are currently unpublished. Eleven of the 27 studies (41\%) performed to date have been to fully define and understand the experimental human ETEC infection model with various ETEC strain/dose combinations. The additional studies were performed to utilize these models to evaluate vaccine candidates $(n=8)$, antibiotics $(n=1)$ or other prophylactic and/or treatment intervention $(n=7)$. Variability in the outcomes reported and types of summary effect estimates utilized was also variable across studies.

Over the past four decades of experimental ETEC infection studies, the diarrhea definition has been quite varied. The first definition utilized by Dupont et al. was "3 watery stools/24-h period" [1]. Subsequently, the diarrhea definition was modified to allow for 
Table 1

List of experimental ETEC infection studies that met inclusion criteria for systematic review and meta-analysis.

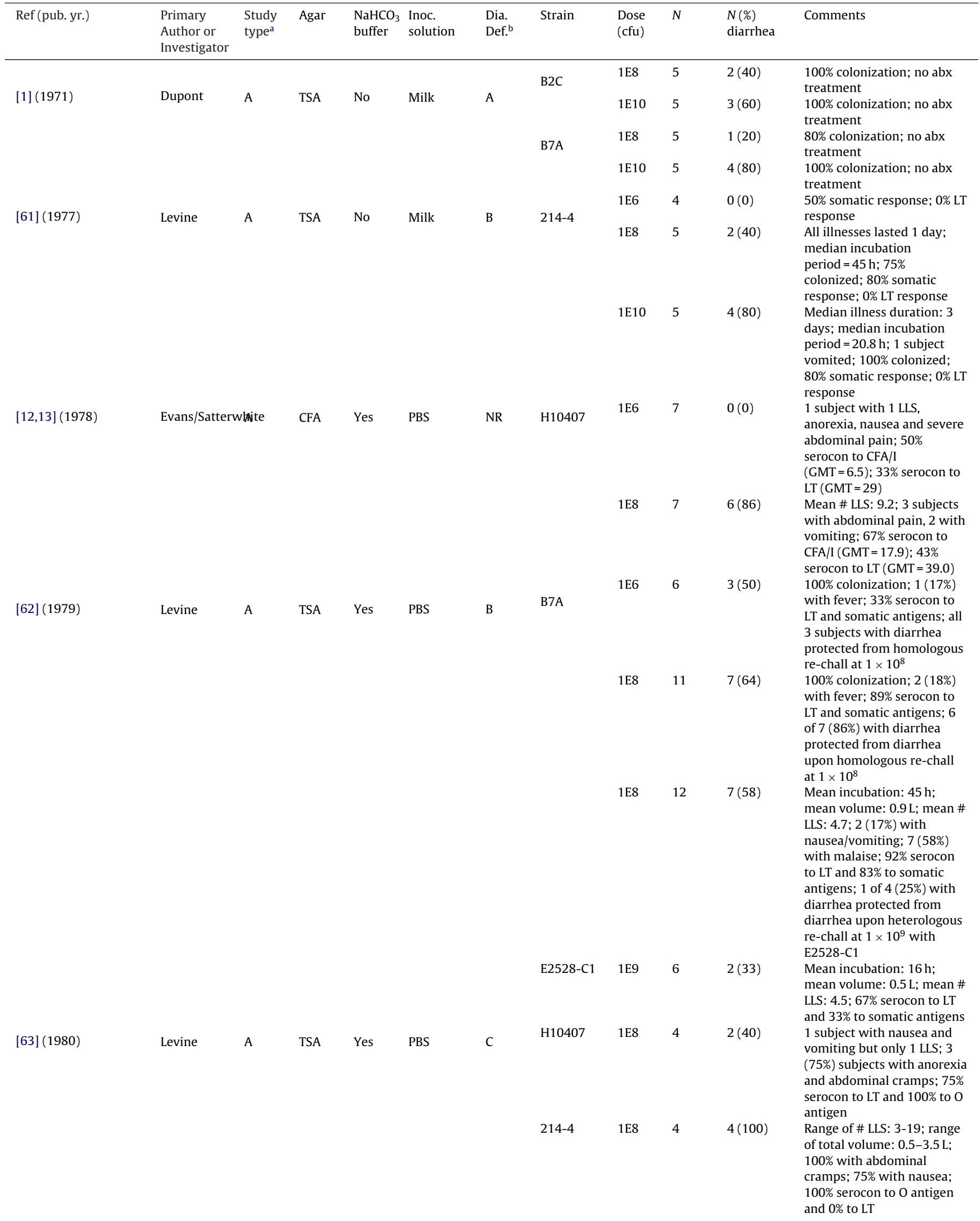


Table 1 (Continued)

\begin{tabular}{|c|c|c|c|c|c|c|c|c|c|c|c|}
\hline $\operatorname{Ref}($ pub. yr.) & $\begin{array}{l}\text { Primary } \\
\text { Author or } \\
\text { Investigator }\end{array}$ & $\begin{array}{l}\text { Study } \\
\text { type }\end{array}$ & Agar & $\begin{array}{l}\mathrm{NaHCO}_{3} \\
\text { buffer }\end{array}$ & $\begin{array}{l}\text { Inoc. } \\
\text { solution }\end{array}$ & $\begin{array}{l}\text { Dia. } \\
\text { Def. }\end{array}$ & Strain & $\begin{array}{l}\text { Dose } \\
\text { (cfu) }\end{array}$ & $N$ & $\begin{array}{l}N(\%) \\
\text { diarrhea }\end{array}$ & Comments \\
\hline \multirow[t]{5}{*}{ [64] (1981) } & \multirow[t]{5}{*}{ Clements } & \multirow[t]{5}{*}{$\mathrm{D}$} & \multirow[t]{5}{*}{ TSA } & \multirow[t]{5}{*}{ Yes } & \multirow[t]{5}{*}{$\mathrm{NaHCO}_{3}$} & \multirow[t]{5}{*}{$\mathrm{D}$} & TD225-C4 & 1E10 & 5 & $2(40)$ & $\begin{array}{l}\text { Mean incubation: } 9.6 \mathrm{~h} \text {; } \\
\text { mean \#LLS: } 3.5 \text {; mean total } \\
\text { volume: } 0.6 \mathrm{~L} \text {; mean } \\
\text { duration: } 18.0 \mathrm{~h} ; 20 \% \text { with } \\
\text { fever, } 0 \% \text { vomiting, } 80 \% \text { with } \\
\text { abd. cramps, } 40 \% \text { with } \\
\text { anorexia, } 60 \% \text { with malaise; } \\
\text { 100\% colonization }\end{array}$ \\
\hline & & & & & & & $214-4$ & $1 \mathrm{E} 8$ & 5 & $4(80)$ & $\begin{array}{l}\text { Mean incubation: } 24.5 \mathrm{~h} \text {; } \\
\text { mean \#LLS: } 5.0 \text {; mean total } \\
\text { volume: } 0.7 \mathrm{~L} \text {; mean } \\
\text { duration: } 30.2 \mathrm{~h} ; 20 \% \text { with } \\
\text { fever, } 0 \% \text { vomiting, } 60 \% \text { with } \\
\text { abd. cramps, } 80 \% \text { with } \\
\text { anorexia, } 80 \% \text { with malaise; } \\
\text { 100\% colonization }\end{array}$ \\
\hline & & & & & & & H10407 & $1 \mathrm{E} 8$ & 4 & $3(75)$ & $\begin{array}{l}\text { Mean incubation: } 57.5 \mathrm{~h} \text {; } \\
\text { mean \#LLS: } 6.7 \text {; mean total } \\
\text { volume: } 1.3 \mathrm{~L} \text {; mean } \\
\text { duration: } 21.3 \mathrm{~h} ; 25 \% \text { with } \\
\text { fever, } 25 \% \text { vomiting, } 100 \% \\
\text { with abd. cramps, } 25 \% \text { with } \\
\text { anorexia, } 0 \% \text { with malaise; } \\
\text { 100\% colonization }\end{array}$ \\
\hline & & & & & & & \multirow[t]{2}{*}{ B7A } & $1 \mathrm{E} 8$ & 3 & $3(100)$ & $\begin{array}{l}\text { Mean incubation: } 23.4 \mathrm{~h} \text {; } \\
\text { mean \#LLS: } 4.7 \text {; mean total } \\
\text { volume: } 0.5 \mathrm{~L} \text {; mean } \\
\text { duration: } 30.2 \mathrm{~h} ; 100 \% \\
\text { colonization }\end{array}$ \\
\hline & & & & & & & & $1 \mathrm{E} 10$ & 8 & $5(63)$ & $\begin{array}{l}\text { Mean incubation: } 14.4 \mathrm{~h} \text {; } \\
\text { mean \#LLS: } 11.8 \text {; mean total } \\
\text { volume: } 1.5 \mathrm{~L} ; \text { mean } \\
\text { duration: } 60.8 \mathrm{~h} ; 100 \% \\
\text { colonization }\end{array}$ \\
\hline [36] (1982) & Black & $\mathrm{C}$ & CFA & Yes & $\mathrm{NaHCO}_{3}$ & $\mathrm{E}$ & H10407 & 5E8-5E9 & 41 & $31(76)$ & $\begin{array}{l}\text { After diarrhea onset, } \\
\text { subjects randomized to } \\
\text { treatment (11 to placebo); } \\
\text { For placebo-treated } \\
\text { subjects: mean duration: } \\
82.1 \text { h; mean \# LLS: } 12.0 ; \\
\text { mean volume: } 2.2 \text { L; } 55 \% \\
\text { vomiting, } 91 \% \text { abd cramps } \\
\text { (lasting } 3.6 \text { days), } 91 \% \\
\text { anorexia (lasting } 3.2 \text { days); } \\
\text { 100\% colonization }\end{array}$ \\
\hline \multirow{4}{*}{ [65] (1982) } & \multirow{4}{*}{ Levine } & \multirow{4}{*}{ B } & \multirow{4}{*}{ CFA } & \multirow{4}{*}{ Yes } & \multirow{4}{*}{ PBS } & \multirow{4}{*}{$\mathrm{D}$} & B7A & 1E10 & 6 & $4(67)$ & $\begin{array}{l}\text { Mean volume: } 0.6 \mathrm{~L} ; \text { mean \# } \\
\text { LLS: } 4.3 ; 100 \% \text { colonization }\end{array}$ \\
\hline & & & & & & & \multirow[t]{3}{*}{ H10407 } & $1 \mathrm{E} 7$ & 11 & $3(27)$ & $\begin{array}{l}\text { Mean volume: } 1.2 \mathrm{~L} \text {; mean \# } \\
\text { LLS: } 15.3 ; 100 \% \text { colonization }\end{array}$ \\
\hline & & & & & & & & $5 \mathrm{E} 8$ & 8 & $7(88)$ & $\begin{array}{l}\text { Mean volume: } 3.0 \mathrm{~L} \text {; mean \# } \\
\text { LLS: } 12.3 ; 100 \% \text { colonization }\end{array}$ \\
\hline & & & & & & & & $5 \mathrm{E} 8$ & 7 & $7(100)$ & $\begin{array}{l}\text { Mean volume: } 4.0 \mathrm{~L} \text {; mean \# } \\
\text { LLS: } 18.0 ; 100 \% \text { malaise and } \\
86 \% \text { vomited; } 2 \text { (29\%) } \\
\text { required IV fluids; } 100 \% \\
\text { colonization }\end{array}$ \\
\hline$[14,15](1983)$ & Graham & $\mathrm{D}$ & CFA & Yes & PBS & $\mathrm{C}$ & H10407 & $2.7 \mathrm{E} 8$ & 16 & $9(56)$ & $\begin{array}{l}\text { Initial phase included } 32 \\
\text { subjects randomized to } \\
\text { prophylaxis with bismuth } \\
\text { subsalicylate }(n=16) \text { or } \\
\text { placebo }(n=16) \text {. After } \\
\text { diarrhea onset, subjects } \\
\text { randomized to treatment } \\
\text { with bismuth subsalicylate } \\
\text { ( } n=6 \text { ) or placebo }(n=5) \text {. For } \\
\text { the } 5 \text { placebo recipients: } \\
\text { mean \# LLS over } 48 \text { h: } 7.0 \text {; } \\
80 \% \text { nausea, } 40 \% \text { vomiting, } \\
100 \% \text { abd cramps, } 40 \% \\
\text { headache, } 60 \% \text { fever }\end{array}$ \\
\hline [66] (1984) & Evans & B & CFA & Yes & PBS & NR & H10407 & $4 \mathrm{E} 8$ & 5 & $2(40)$ & $\begin{array}{l}\text { No data on additional } \\
\text { ETEC-associated symptoms } \\
\text { provided }\end{array}$ \\
\hline
\end{tabular}


Table 1 (Continued)

\begin{tabular}{|c|c|c|c|c|c|c|c|c|c|c|c|}
\hline Ref (pub. yr.) & $\begin{array}{l}\text { Primary } \\
\text { Author or } \\
\text { Investigator }\end{array}$ & $\begin{array}{l}\text { Study } \\
\text { type }^{\mathrm{a}}\end{array}$ & Agar & $\begin{array}{l}\mathrm{NaHCO}_{3} \\
\text { buffer }\end{array}$ & $\begin{array}{l}\text { Inoc. } \\
\text { solution }\end{array}$ & $\begin{array}{l}\text { Dia. } \\
\text { Def. }\end{array}$ & Strain & $\begin{array}{l}\text { Dose } \\
\text { (cfu) }\end{array}$ & $N$ & $\begin{array}{l}N(\%) \\
\text { diarrhea }\end{array}$ & Comments \\
\hline & & & & & & & H1765 & $4 \mathrm{E} 8$ & 6 & $5(83)$ & $\begin{array}{l}\text { No data on additional } \\
\text { ETEC-associated symptoms } \\
\text { provided }\end{array}$ \\
\hline [67] (1984) & Levine & A & TSA & Yes & PBS & NR & E24377A & $5 \mathrm{E} 8$ & 14 & $9(64)$ & $\begin{array}{l}50 \% \text { seroconversion to CS1 } \\
\text { and } 50 \% \text { to CS3; No data on } \\
\text { additional ETEC-associated } \\
\text { symptoms provided }\end{array}$ \\
\hline$[16,17](1986)$ & Levine & B & NR & NR & NR & NR & E24377A & $5 \mathrm{E} 8$ & 6 & $6(100)$ & $\begin{array}{l}\text { Study published in } 2 \text { book } \\
\text { chapters; no additional data } \\
\text { provided }\end{array}$ \\
\hline [30] (1988) & Evans & B & CFA & Yes & PBS & $\mathrm{F}$ & H10407 & $5 \mathrm{E} 9$ & 5 & $5(100)$ & $\begin{array}{l}\text { Mean stool weight at peak } \\
\text { of illness: } 765 \mathrm{~g} \text {; }\end{array}$ \\
\hline [31] (1988) & Evans & B & CFA & Yes & PBS & $\mathrm{F}$ & H10407 & $5 \mathrm{E} 9$ & 9 & $8(89)$ & $\begin{array}{l}\text { Mean total weight for } 24 \mathrm{~h} \\
\text { during peak illness: } 900 \mathrm{~g} \text {; } \\
\text { mean time to 1st LLS: } \\
30.9 \mathrm{~h} ; 88 \% \text { colonization }\end{array}$ \\
\hline [40] (1988) & Tacket & $\mathrm{D}$ & NR & Yes & $\mathrm{NaHCO}_{3}$ & G & H10407 & $1.2 \mathrm{E} 9$ & 10 & $9(90)$ & $\begin{array}{l}\text { Subjects received } \mathrm{NaHCO}_{3} \\
\text { buffer over } 2 \text { days prior to } \\
\text { and for } 5 \text { days after ETEC } \\
\text { admin as part of } \\
\text { immunoprophylaxis. Mean } \\
\text { diarrhea volume: } 1.5 \mathrm{~L} \text {; } \\
\text { mean diarrhea onset time: } \\
48 \text { h; } 80 \% \text { abdominal } \\
\text { cramps, } 60 \% \text { vomiting, } 90 \% \\
\text { malaise, } 20 \% \text { fever, } 90 \% \\
\text { anorexia; } 60 \% \text { received early } \\
\text { abx.; } 100 \% \text { colonization; } 40 \% \\
\text { seroconversion to CFA/I, 50\% } \\
\text { to LT and } 100 \% \text { to O antigen }\end{array}$ \\
\hline [68] (1994) & Tacket & B & NR & Yes & $\mathrm{NaHCO}_{3}$ & NR & E24377A & 3E9 & 10 & $10(100)$ & $\begin{array}{l}\text { Mean volume: } 1.5 \mathrm{~L} \text {; mean \# } \\
\text { LLS: } 8.6 ; 90 \%, 40 \% \text { and } 90 \% \\
\text { IGA ASC responses to CFA/II, } \\
\text { CS1 and CS3, respectively }\end{array}$ \\
\hline [41] (1998) & Freedman & $\mathrm{D}$ & NR & Yes & $\mathrm{NaHCO}_{3}$ & G & H10407 & $1 \mathrm{E} 9$ & 10 & $7(70)$ & $\begin{array}{l}\text { Subjects received } \mathrm{NaHCO}_{3} \\
\text { buffer over } 2 \text { days prior to } \\
\text { and for } 5 \text { days after ETEC } \\
\text { admin as part of } \\
\text { immunoprophylaxis. Mean } \\
\text { volume: } 1.3 \mathrm{~L} \text {; mean \# LLS: } \\
7.4 ; 100 \% \text { abd cramps, } 60 \% \\
\text { anorexia, } 50 \% \text { headache, } 30 \% \\
\text { malaise; } 100 \% \text { serologic } \\
\text { response to CFA/I, LPS and LT }\end{array}$ \\
\hline [69] (1999) & Tacket & $\mathrm{D}$ & NR & No & $\begin{array}{l}\text { Apple- } \\
\text { sauce }\end{array}$ & G & E24377A & $1 \mathrm{E} 8$ & 10 & $3(30)$ & $\begin{array}{l}\text { Mean volume: } 0.8 \mathrm{~L} \text {; mean \# } \\
\text { LLS: } 5.3 ; 100 \% \text { colonization; } \\
\text { 100\% seroconversion to LT } \\
\text { and } 20 \% \text { to CS3 }\end{array}$ \\
\hline \multirow[t]{3}{*}[70]{$(2007)^{c}$} & \multirow[t]{3}{*}{ Coster } & \multirow[t]{3}{*}{ A } & \multirow[t]{3}{*}{ TSA } & \multirow[t]{3}{*}{ Yes } & \multirow[t]{3}{*}{$\mathrm{NaHCO}_{3}$} & \multirow[t]{3}{*}{ C } & B7A & $1.5 \mathrm{E} 9$ & 8 & $5(63)$ & $\begin{array}{l}\text { Mean weight: } 1.0 \mathrm{~kg} \text {; mean } \\
\text { onset time: } 10 \mathrm{~h} ; 25 \% \\
\text { mod-sev abd cramps, } 25 \% \\
\text { mod-sev headache, } 13 \% \\
\text { mod-sev nausea, } 13 \% \\
\text { mod-sev loss of appetite, } 0 \% \\
\text { mod-sev fever; } 100 \% \\
\text { colonization }\end{array}$ \\
\hline & & & & & & & & $1.4 \mathrm{E} 10$ & 8 & $8(100)$ & $\begin{array}{l}\text { Mean weight: } 0.9 \mathrm{~kg} ; \text { mean } \\
\text { onset time: } 12 \mathrm{~h} ; 38 \% \\
\text { mod-sev abd cramps, } 38 \% \\
\text { mod-sev headache, } 38 \% \\
\text { mod-sev nausea, } 0 \% \\
\text { mod-sev loss of appetite, } \\
\text { 25\% mod-sev fever; } 100 \% \\
\text { colonization }\end{array}$ \\
\hline & & & & & & & H10407 & $1.2 \mathrm{E} 8$ & 7 & $6(86)$ & $\begin{array}{l}\text { Mean weight: } 1.9 \mathrm{~kg} ; \text { mean } \\
\text { onset time: } 43 \mathrm{~h} ; 71 \% \\
\text { mod-sev abd cramps, } 57 \% \\
\text { mod-sev headache, } 43 \% \\
\text { mod-sev nausea, } 71 \% \\
\text { mod-sev loss of appetite, } \\
\text { 43\% mod-sev fever; } 100 \% \\
\text { colonization }\end{array}$ \\
\hline
\end{tabular}


Table 1 (Continued)

\begin{tabular}{|c|c|c|c|c|c|c|c|c|c|c|c|}
\hline Ref (pub. yr.) & $\begin{array}{l}\text { Primary } \\
\text { Author or } \\
\text { Investigator }\end{array}$ & $\begin{array}{l}\text { Study } \\
\text { type }^{\mathrm{a}}\end{array}$ & Agar & $\begin{array}{l}\mathrm{NaHCO}_{3} \\
\text { buffer }\end{array}$ & $\begin{array}{l}\text { Inoc. } \\
\text { solution }\end{array}$ & $\begin{array}{l}\text { Dia. } \\
\text { Def. }\end{array}$ & Strain & $\begin{array}{l}\text { Dose } \\
\text { (cfu) }\end{array}$ & $N$ & $\begin{array}{l}N(\%) \\
\text { diarrhea }\end{array}$ & Comments \\
\hline & & & & & & & & $1.4 \mathrm{E} 9$ & 8 & $7(88)$ & $\begin{array}{l}\text { Mean weight: } 1.9 \mathrm{~kg} ; \text { mean } \\
\text { onset time: } 34 \text { h; } 100 \% \\
\text { mod-sev abd cramps, } 63 \% \\
\text { mod-sev headache, } 63 \% \\
\text { mod-sev nausea, } 75 \% \\
\text { mod-sev loss of appetite, } \\
\text { 38\% mod-sev fever; } 100 \% \\
\text { colonization }\end{array}$ \\
\hline [21] (2007) & McKenzie & B & CFA & Yes & $\mathrm{NaHCO}_{3}$ & G & E24377A & $6 \mathrm{E} 8$ & 20 & $20(100)$ & $\begin{array}{l}\text { Mean weight: } 1.1 \mathrm{~kg} \text {; mean } \\
\text { \# LLS: } 9.7 ; 40 \% \text { received IV } \\
\text { fluids; mean time to } \\
\text { diarrhea onset: } 29 \mathrm{~h} \text {; mean } \\
\text { time to abx: } 51 \mathrm{~h}\end{array}$ \\
\hline 2008 [71] & McKenzie & B & CFA & Yes & $\mathrm{NaHCO}_{3}$ & G & E24377A & 3E9 & 16 & $13(81)$ & $\begin{array}{l}\text { Median volume: } 0.9 \mathrm{~L} ; \\
\text { median \# LLS: } 6 \text {; median } \\
\text { max. } 24 \mathrm{~h} \text {, volume: } 0.9 \mathrm{~L} ; \\
\text { median time to diarrhea } \\
\text { onset: } 24.1 \mathrm{~h} \text {; median } \\
\text { duration: } 32.2 \mathrm{~h} ; 0 \% \text { fever, } \\
\text { 67\% malaise; } 73 \% \text { abdominal } \\
\text { cramps; } 47 \% \text { nausea; } 33 \% \\
\text { headache; } 7 \% \text { vomiting; } 31 \% \\
\text { IV fluids; } 50 \% \text { early abx }\end{array}$ \\
\hline Unpub $^{c}$ & McKenzie & A & CFA & Yes & $\mathrm{NaHCO}_{3}$ & G & H10407 & $1.1 \mathrm{E} 9$ & 5 & $5(100)$ & $\begin{array}{l}\text { Median volume: } 1.5 \mathrm{~L} \text {; } \\
\text { median \# LLS: } 13 \text {; median } \\
\text { max. } 24 \text { h, volume: } 1.0 \mathrm{~L} ; \\
\text { median time to diarrhea } \\
\text { onset: } 25.7 \mathrm{~h} \text {; median } \\
\text { duration: } 46.6 \mathrm{~h} ; 0 \% \text { fever, } \\
\text { 80\% malaise; } 80 \% \text { abdominal } \\
\text { cramps; } 60 \% \text { nausea; } 80 \% \\
\text { headache; } 40 \% \text { vomiting; } \\
\text { 20\% IV fluids; } 100 \% \text { early abx }\end{array}$ \\
\hline Unpub $^{c}$ & McKenzie & $\mathrm{D}$ & CFA & Yes & $\mathrm{NaHCO}_{3}$ & G & H10407 & $1 \mathrm{E} 9$ & 11 & $9(82)$ & $\begin{array}{l}\text { Subjects received } \mathrm{NaHCO}_{3} \\
\text { buffer over } 2 \text { days prior to } \\
\text { and for } 3 \text { days after ETEC } \\
\text { admin as part of } \\
\text { immunoprophylaxis. } \\
\text { Median volume: } 1.9 \mathrm{~L} \text {; } \\
\text { median \# LLS: } 10 ; \text { median } \\
\text { max. } 24 \text { h, volume: } 1.2 \mathrm{~L} \text {; } \\
\text { median time to diarrhea } \\
\text { onset: } 22.8 \mathrm{~h} \text {; median } \\
\text { duration: } 53.8 \text { h; } 27 \% \text { fever, } \\
55 \% \text { malaise; } 73 \% \text { abdominal } \\
\text { cramps; } 36 \% \text { nausea; } 73 \% \\
\text { headache; } 18 \% \text { vomiting; } \\
45 \% \text { IV fluids; } 55 \% \text { early abx }\end{array}$ \\
\hline \multirow[t]{2}{*}[72]{$^{c}$} & McKenzie & A & CFA & Yes & $\mathrm{NaHCO}_{3}$ & G & $\begin{array}{l}\text { LSN03- } \\
\text { 016011/A }\end{array}$ & $7.0 \mathrm{E} 8$ & 5 & $3(60)$ & $\begin{array}{l}\text { Median volume: } 0.8 \mathrm{~L} ; \\
\text { median \# LLS: } 6 \text {; median } \\
\text { max. } 24 \text { h, volume: } 0.6 \mathrm{~L} \text {; } \\
\text { median time to diarrhea } \\
\text { onset: } 11.7 \mathrm{~h} \text {; median } \\
\text { duration: } 27.3 \mathrm{~h} \text {; } 0 \% \text { fever, } \\
\text { 60\% malaise; } 80 \% \text { abdominal } \\
\text { cramps; } 20 \% \text { nausea; } 20 \% \\
\text { headache; } 40 \% \text { vomiting; } \\
\text { 20\% IV fluids; } 60 \% \text { early abx }\end{array}$ \\
\hline & & & & & & & & $6.2 \mathrm{E} 9$ & 8 & $7(88)$ & $\begin{array}{l}\text { Median volume: } 1.0 \mathrm{~L} ; \\
\text { median \# LLS: } 6 \text {; median } \\
\text { max. } 24 \text { h, volume: } 0.5 \mathrm{~L} ; \\
\text { median time to diarrhea } \\
\text { onset: } 11.7 \mathrm{~h} \text {; median } \\
\text { duration h: } 10.4 ; 0 \% \text { fever, } \\
63 \% \text { malaise; } 75 \% \text { abdominal } \\
\text { cramps; } 25 \% \text { nausea; } 25 \% \\
\text { headache; } 25 \% \text { vomiting; } \\
\text { 13\% IV fluids; } 38 \% \text { early abx }\end{array}$ \\
\hline
\end{tabular}


Table 1 (Continued)

\begin{tabular}{|c|c|c|c|c|c|c|c|c|c|c|c|}
\hline Ref (pub. yr.) & $\begin{array}{l}\text { Primary } \\
\text { Author or }\end{array}$ & $\begin{array}{l}\text { Study } \\
\text { type }^{\text {a }}\end{array}$ & Agar & $\begin{array}{l}\mathrm{NaHCO}_{3} \\
\text { buffer }\end{array}$ & $\begin{array}{l}\text { Inoc. } \\
\text { solution }\end{array}$ & $\begin{array}{l}\text { Dia. } \\
\text { Def. }\end{array}$ & Strain & $\begin{array}{l}\text { Dose } \\
\text { (cfu) }\end{array}$ & $N$ & $\begin{array}{l}N(\%) \\
\text { diarrhea }\end{array}$ & Comments \\
\hline
\end{tabular}

$[72]^{c}$

Investigator

$[72]^{c}$

McKenzie D

CFA Yes $\quad \mathrm{NaHCO}_{3}$

G

LSN03-
$016011 / A$
016011/A

5E9 $12 \quad 6(50)$


Table 1 (Continued)

\begin{tabular}{|c|c|c|c|c|c|c|c|c|c|c|c|}
\hline Ref (pub. yr.) & $\begin{array}{l}\text { Primary } \\
\text { Author or } \\
\text { Investigator }\end{array}$ & $\begin{array}{l}\text { Study } \\
\text { type }^{a}\end{array}$ & Agar & $\begin{array}{l}\mathrm{NaHCO}_{3} \\
\text { buffer }\end{array}$ & $\begin{array}{l}\text { Inoc. } \\
\text { solution }\end{array}$ & $\begin{array}{l}\text { Dia. } \\
\text { Def. }^{b}\end{array}$ & Strain & $\begin{array}{l}\text { Dose } \\
\text { (cfu) }\end{array}$ & $N$ & $\begin{array}{l}N(\%) \\
\text { diarrhea }\end{array}$ & Comments \\
\hline & & & & & & & & $4.9 \mathrm{E} 9$ & 5 & $4(80)$ & $\begin{array}{l}\text { Median volume: } 0.7 \mathrm{~L} \text {; } \\
\text { median \# LLS: 7; median } \\
\text { max. } 24 \text { h, volume: } 0.7 \mathrm{~L} ; \\
\text { median time to diarrhea } \\
\text { onset: } 22.5 \mathrm{~h} \text {; median } \\
\text { duration: } 29.8 \mathrm{~h} ; 0 \% \text { fever, } \\
\text { 40\% malaise; } 60 \% \text { abdominal } \\
\text { cramps; } 60 \% \text { nausea; } 40 \% \\
\text { headache; } 20 \% \text { vomiting; } \\
20 \% \text { IV fluids; } 40 \% \text { early abx }\end{array}$ \\
\hline
\end{tabular}

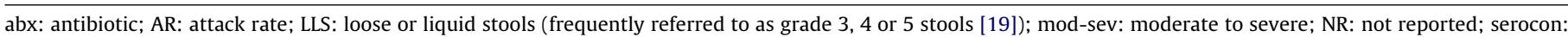
seroconversion; \#: number.

a Study types: (A) pathogenesis; (B) vaccine efficacy; (C) antibiotic treatment; (D) other treatment and/or prophylaxis.

b Diarrhea definitions: (A) $\geq 3$ LLS in $24 \mathrm{~h}$; (B) $\geq 3$ LLS in $24 \mathrm{~h}$ or $1 \mathrm{LLS} \geq 200 \mathrm{~mL}$; (C) $\geq 2$ LLS in $24 \mathrm{~h}$; (D) $\geq 3$ LLS or $\geq 2 \mathrm{LLS}$ at $\geq 200 \mathrm{~mL}$ in $48 \mathrm{~h}$ or $1 \mathrm{LLS} \geq 300 \mathrm{~mL}$; (E) $\geq 2 \mathrm{LLS}$; (F) $\geq 2$ LLS +1 somatic complaint; $(G) \geq 2$ LLS in $48 \mathrm{~h}$ at $\geq 200 \mathrm{~mL}$ or $1 \mathrm{LLS} \geq 300 \mathrm{~mL}$.

c These studies used ETEC strains for which the seed lots were manufactured under Good Manufacturing Practices.

a volume quantification of either 1 or 2 loose stools over a 24 or $48 \mathrm{~h}$ time period. In 1978, Levine et al. established a grading system for scoring stools which has been subsequently used to classify loose stools as those coded as grade 3 ("thick liquid"), 4 ("opaque-watery") or 5 ("rice-water") [19]. This grading system has been consistently utilized since, although the number and/or quantity of stools required to meet the diarrhea definition remained inconsistent. The most common diarrhea outcome definition, used in $41 \%$ of the studies is $\geq 200 \mathrm{~mL}$ of Grade 3, 4 or 5 stools within a $48 \mathrm{~h}$ period or 1 Grade 3,4 or 5 stool totaling $\geq 300 \mathrm{~mL}$.

Eleven different strains have been utilized in these studies and summary information about each is provided in Table 2 . The three most commonly administered strains are H10407, E24377A and B7A all three of which express both the LT and ST enterotoxins. As shown in Fig. 2, the percent of subjects reaching the primary outcome of diarrhea has varied within and across a range of dose-strain combinations. The majority of studies (88.5\%) involved volunteer pretreatment with sodium bicarbonate prior to administration of the challenge inoculum. Most commonly the vehicle to administer the challenge strain was also sodium bicarbonate (52\%), although saline (33\%), milk (7\%) and apple sauce (4\%) have also been used.

When limiting our analyses to the 3 most utilized strains, B7A, H10407 and E24377A, we found that the diarrhea attack rate was dose-dependent with increasing doses associated with higher attack rates $(p<0.01)$. Interestingly, when evaluating these strains at doses of $5 \times 10^{8}$ and higher $\mathrm{cfu}$, there was no difference in diarrhea attack rates (heterogeneity chi-square $p=0.07$ ) across any of the strains at doses up to $1 \times 10^{10}$, with an overall attack rate of $87 \%$ (95\% CI 82, 92) and strain-specific attack rates of $78 \%$ (95\% CI: 64 , 92), 89\% (95\% CI: 79, 98) and 87\% (95\% CI: 81, 93) for B7A, E24377A

Table 2

Detailed information on strains of ETEC that have used for experimental human infection.

\begin{tabular}{|c|c|c|c|c|c|c|c|}
\hline Strain name & Initial strain description & Serotype & $\mathrm{CF}(\mathrm{s})$ & Toxin(s) & Country/region of origin & $\begin{array}{l}\text { Clinical information on index } \\
\text { case }\end{array}$ & $\begin{array}{l}\text { Range of } \\
\text { doses } \\
\text { administered }\end{array}$ \\
\hline $214-4$ & [73] & Not typeable & Unknown & ST & Mexico & $\begin{array}{l}\text { Isolated from } 29 \text { year-old } \\
\text { Caucasian male physician with } \\
\text { travelers' diarrhea } \\
\text { characterized by watery } \\
\text { diarrhea, abdominal cramps, } \\
\text { malaise, nausea and fever }\end{array}$ & 1E6-1E10 \\
\hline $\mathrm{B} 2 \mathrm{C}$ & [1] & O6:H16 & $\mathrm{CS} 2, \mathrm{CS} 3$ & $\mathrm{LT} / \mathrm{ST}$ & Vietnam & $\begin{array}{l}\text { Diarrhea case in US military } \\
\text { adult }\end{array}$ & $1 \mathrm{E} 8$ \\
\hline B7A & [1] & 0148:H28 & CS6 & LT/ST & Vietnam & $\begin{array}{l}\text { Diarrhea case in US military } \\
\text { adult serving in Vietnam }\end{array}$ & $1 \mathrm{E} 6-1 \mathrm{E} 10$ \\
\hline DS26-1 & {$[72]$} & O8:H9 & CS19 & LT & Saudi Arabia & $\begin{array}{l}\text { Isolated in } 1990 \text { from a U.S. } \\
\text { soldier with diarrhea while on } \\
\text { deployment during Operation } \\
\text { Desert Shield }\end{array}$ & $5 \mathrm{E} 8$ \\
\hline E24377A & [74] & 0139:H28 & $\mathrm{CS} 1, \mathrm{CS} 3$ & $\mathrm{LT} / \mathrm{ST}$ & Egypt & $\begin{array}{l}\text { Traveler returning from Egypt } \\
\text { with ETEC disease }\end{array}$ & 1E8-3E9 \\
\hline E2528-C1 & {$[75]$} & O25:NM & Unknown & LT & Caribbean & Cruise ship diarrhea outbreak & $1 \mathrm{E} 9$ \\
\hline H10407 & {$[76]$} & O78:K80:H11 & CFA/I & $\mathrm{LT} / \mathrm{ST}$ & Bangladesh & Severe case of watery diarrhea & 1E6-5E9 \\
\hline H1765 & {$[66]$} & O6:K15:H16 & CFA/II & $\mathrm{LT} / \mathrm{ST}$ & Bangladesh & Unknown & $4 \mathrm{E} 8$ \\
\hline LSN03-016011/A & {$[72]$} & O8:H- & CS17 & LT & Turkey & $\begin{array}{l}\text { Isolated from } 29 \text { year-old U.S. } \\
\text { female military with acute, } \\
\text { watery diarrhea shortly after } \\
\text { arrival at Incirlik Airbase in } \\
\text { Turkey }\end{array}$ & 5E8-5E9 \\
\hline TD225-C4 & [64] & O75:H9 & Unknown & LT & Mexico & Case of diarrhea & $1 \mathrm{E} 10$ \\
\hline WS0115A & [77] & 0114:H- & CS19 & $\mathrm{LT} / \mathrm{ST}$ & Egypt & $\begin{array}{l}\text { Isolated from stool of } \\
12 \text {-month old Egyptian female } \\
\text { suffering from watery diarrhea } \\
\text { in Abees, Egypt }\end{array}$ & 5E8-5E10 \\
\hline
\end{tabular}




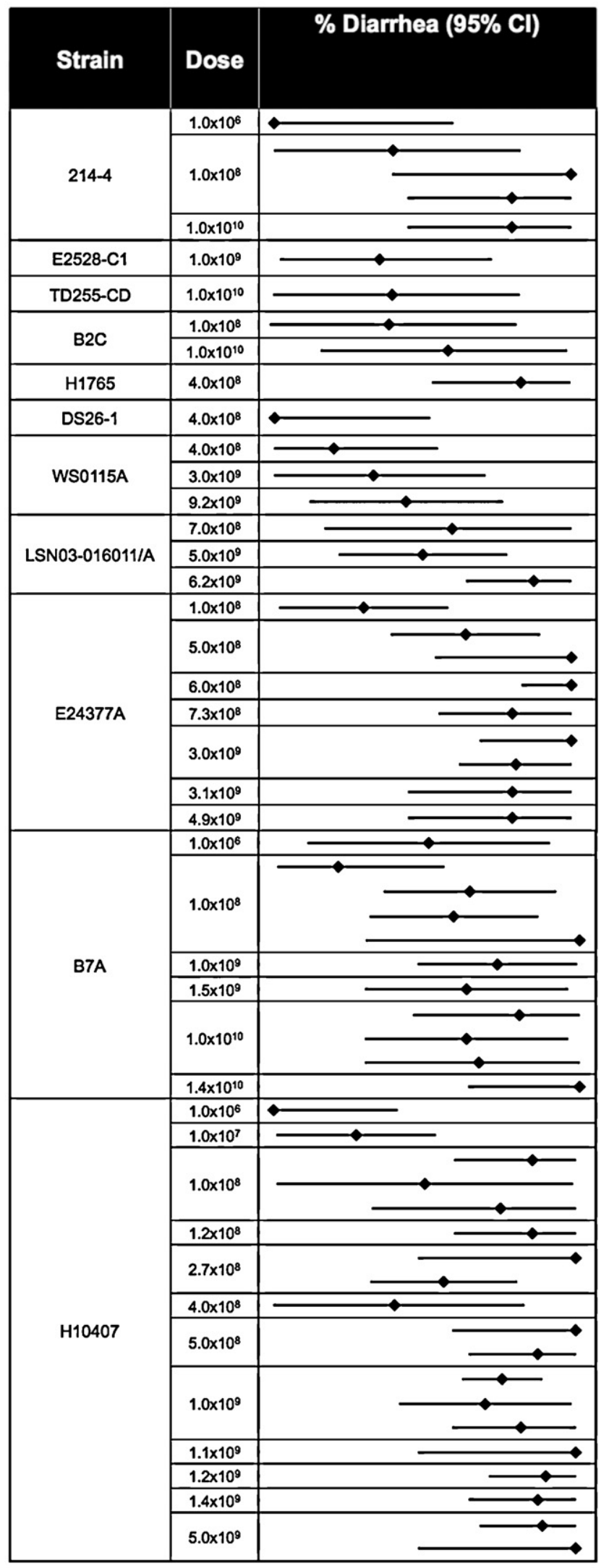

Fig. 2. Point estimate and $95 \%$ confidence interval for the proportion of subjects meeting the primary outcome of diarrhea for all included dose and strain combinations. Diamond: point estimate; horizontal line: 95\% confidence interval. and H10407, respectively. The diarrhea attack rates of strains with similar toxin phenotypes at the $\geq 5 \mathrm{E} 8$ dose showed significant heterogeneity, with the highest rates following administration of LT/ST strains (84\%; 95\% confidence interval $\{\mathrm{CI}\}: 78,89$; heterogeneity chi-square $p=0.02$ ) followed by ST only strains ( $80 \%$; $95 \%$ CI: 52 , 100 ; no $p$-value as only 1 study included) and LT only strains (57\%; $95 \% \mathrm{CI}: 33,81$; heterogeneity chi-square $p=0.02$ ). For the purposes of this analysis, we excluded the DS26-1 strain which did not induce diarrhea at any of the inoculum doses administered. We found no significant effect on diarrhea rates with changing inoculum solution, agar used for strain growth or fasting time, although our power was relatively limited to find these effects.

\subsection{Individual patient level data}

\subsubsection{Clinical data}

The individual patient-level data is shown in Table 3 and includes 133 subjects administered 1 of 6 ETEC strains at doses of $1 \mathrm{E} 8$ to $1 \mathrm{E} 10$. The highest diarrhea attack rates were seen with B7A at $1 \mathrm{E} 10 \mathrm{cfu}(100.0 \%), \mathrm{H} 10407$ at $1 \mathrm{E} 9 \mathrm{cfu}(87.5 \%)$ and E24377A at $\sim 5 E 9 \mathrm{cfu}(80.8 \%)$. Strains expressing CS17 (LSN03-016011/A) and CS19 (WS0115A) also caused diarrhea, although at lower attack rates and a strain expressing CS19 (DS26-1) caused no diarrhea. There was a higher proportion of severe diarrhea among all doses of H10407 compared to the other strains. The only dose/strain combination that resulted in a similar proportion of severe diarrhea was the B7A strain at the 1E10 inoculum.

There were also apparent strain differences in the nondiarrheal symptoms following ETEC inoculation. While abdominal cramps/pain, nausea, malaise and headache were relatively common across all doses and strains (excluding DS26-1), vomiting was seen most frequently following inoculation with the strains H10407, B7A and LSN03-016011/A. Similarly, fever was relatively infrequent in all strains except $\mathrm{H} 10407$ and B7A. While most strains demonstrated a range of severity in these ETEC-associated symptoms, the H10407 strain exhibited markedly more severe symptoms. The need for intravenous fluids (I.V.) was relatively rare, possibly due to the high frequency of early antibiotic treatment across dose/strain combinations. Although not shown here, all subjects were encouraged to consume oral rehydration solution or other oral fluids at diarrheal onset, potentially decreasing the need for I.V. fluids.

A more detailed description of the diarrhea episode among subjects meeting the diarrhea definition is shown in Table 4. Regardless of dose, H10407 exhibited a significantly higher number of unformed stools (all $p<0.013$ except: B7A $\{p=0.014\}$, E24377A $\{p=0.022\}$ ), total volume (all $p<0.013$ ) and maximum $24 \mathrm{~h}$ volume (all $p<0.013$ except: E24377A $\{p=0.022\}$ ) than the other strains. The increase in the total number of unformed stools and total diarrhea may be explained by the longer duration of the diarrheal episode seen with both evaluated doses of the H10407 strain. Interestingly, the time to antibiotic treatment was quite variable across dose/strain combinations, likely reflecting differences in strain pathogenicity and clinical treatment algorithms.

The median time to the first loose stool was quite variable, and ranged from 6.8 to $50.0 \mathrm{~h}$ post-inoculation. Fig. 3 shows a detailed representation of stool volume accumulation and number of stools for the four strains resulting in the highest diarrhea attack rates. The volume of loose stools at the highest H10407 dose $\left(1 \times 10^{9}\right)$ increases at a higher rate than the other strains regardless of dose, and continues to increase throughout the observation period. In contrast, the output for the 'low dose' $\left(1 \times 10^{8}\right)$ of H10407 is relatively consistent with the other dose/strain combinations until the $72 \mathrm{~h}$ timepoint when it increases remarkably. The stool volume and count for the 'low dose' $\left(5 \times 10^{8}\right)$ of strain E24377A falls between 
Table 3

Description of primary outcomes following experimental infection with enterotoxigenic Escherichia coli (ETEC)

\begin{tabular}{|c|c|c|c|c|c|c|c|c|c|c|c|c|}
\hline \multirow{2}{*}{$\begin{array}{l}\text { Strain } \\
\text { Approximate dose }\end{array}$} & \multicolumn{2}{|l|}{ H10407 } & \multicolumn{2}{|l|}{ E24377A } & \multicolumn{2}{|l|}{$\mathrm{B} 7 \mathrm{~A}$} & \multicolumn{2}{|c|}{ LSN03-016011/A } & \multicolumn{2}{|l|}{ WS0115A } & \multicolumn{2}{|l|}{ DS26-1 } \\
\hline & $1 \mathrm{E} 8$ & $1 \mathrm{E} 9$ & $5 \mathrm{E} 8$ & $5 \mathrm{E} 9$ & $1 \mathrm{E} 9$ & $1 \mathrm{E} 10$ & $5 \mathrm{E} 8$ & $5 \mathrm{E} 9$ & $5 \mathrm{E} 8$ & $5 \mathrm{E} 9$ & $1 \mathrm{E} 10$ & $5 \mathrm{E} 8$ \\
\hline$N$ & 7 & 24 & 10 & 26 & 8 & 8 & 5 & 20 & 5 & 6 & 9 & 5 \\
\hline$\%$ male & 57.1 & 58.3 & 90.0 & 76.9 & 87.5 & 50.0 & 80.0 & 60.0 & 60.0 & 66.7 & 88.9 & 60.0 \\
\hline \% African American & 42.9 & 70.8 & 80.0 & 76.9 & 50.0 & 37.5 & 100.0 & 85.0 & 100.0 & 100.0 & 88.9 & 100.0 \\
\hline Median (IQR) age & $\begin{array}{l}46.2(37.4 \\
49.6)\end{array}$ & $\begin{array}{l}31.6(25.4 \\
40.3)\end{array}$ & $\begin{array}{l}43.0(21.0, \\
47.0)\end{array}$ & $\begin{array}{l}37.5(22.0 \\
43.0)\end{array}$ & $\begin{array}{l}43.2(25.6 \\
51.2)\end{array}$ & $\begin{array}{l}37.2(28.1 \\
43.8)\end{array}$ & $\begin{array}{l}34.9(32.9 \\
35.8)\end{array}$ & $\begin{array}{l}31.6(25.1 \\
41.1)\end{array}$ & $\begin{array}{l}40.3(38.6 \\
44.5)\end{array}$ & $\begin{array}{l}34.3(24.8 \\
41.3)\end{array}$ & $\begin{array}{l}25.5(24.0, \\
31.2)\end{array}$ & $\begin{array}{l}28.7(19.8 \\
30.3)\end{array}$ \\
\hline \% diarrhea & 85.7 & 87.5 & 70.0 & 80.8 & 62.5 & 100.0 & 60.0 & 65.0 & 20.0 & 33.3 & 44.4 & 0.0 \\
\hline Mild & 0 & 12.5 & 20.0 & 19.2 & 25.0 & 0.0 & 20.0 & 15.0 & 0.0 & 0.0 & 0.0 & 0.0 \\
\hline Moderate & 0 & 4.2 & 30.0 & 23.1 & 0.0 & 25.0 & 0.0 & 30.0 & 20.0 & 33.3 & 33.3 & 0.0 \\
\hline Severe & 85.7 & 70.8 & 20.0 & 38.5 & 37.5 & 75.0 & 40.0 & 20.0 & 0.0 & 0.0 & 11.1 & 0.0 \\
\hline \% abdominal cramps & 85.7 & 83.3 & 62.5 & 76.0 & 62.5 & 62.5 & 80.0 & 60.0 & 40.0 & 50.0 & 22.2 & 0.0 \\
\hline Mild & 14.3 & 16.7 & 25.0 & 28.0 & 37.5 & 25.0 & 0.0 & 10.0 & 40.0 & 0.0 & 11.1 & 0.0 \\
\hline Moderate & 0 & 29.2 & 37.5 & 24.0 & 12.5 & 0.0 & 20.0 & 30.0 & 0.0 & 50.0 & 11.1 & 0.0 \\
\hline Severe & 71.4 & 37.5 & 0.0 & 24.0 & 12.5 & 37.5 & 60.0 & 20.0 & 0.0 & 0.0 & 0.0 & 0.0 \\
\hline \% Nausea & 71.4 & 50.0 & 25.0 & 48.0 & 37.5 & 62.5 & 20.0 & 35.0 & 20.0 & 33.3 & 11.1 & 0.0 \\
\hline Mild & 28.6 & 4.2 & 25.0 & 24.0 & 25.0 & 25.0 & 0.0 & 65.0 & 20.0 & 16.7 & 16.7 & 0.0 \\
\hline Moderate & 0 & 8.3 & 0.0 & 12.0 & 12.5 & 0.0 & 0.0 & 20.0 & 0.0 & 0.0 & 0.0 & 0.0 \\
\hline Severe & 42.9 & 37.5 & 0.0 & 12.0 & 0.0 & 37.5 & 20.0 & 15.0 & 0.0 & 16.7 & 0.0 & 0.0 \\
\hline$\%$ Malaise & 57.1 & 62.5 & 50.0 & 64.0 & 0.0 & 37.5 & 60.0 & 45.0 & 20.0 & 50.0 & 22.2 & 0.0 \\
\hline Mild & 0 & 8.3 & 12.5 & 20.0 & 0.0 & 12.5 & 0.0 & 20.0 & 20.0 & 0.0 & 22.2 & 0.0 \\
\hline Moderate & 14.3 & 25 & 25.0 & 24.0 & 0.0 & 12.5 & 20.0 & 10.0 & 0.0 & 16.7 & 0.0 & 0.0 \\
\hline Severe & 42.9 & 29.2 & 12.5 & 20.0 & 0.0 & 12.5 & 40.0 & 15.0 & 0.0 & 33.3 & 0.0 & 0.0 \\
\hline$\%$ headache & 57.1 & 70.8 & 12.5 & 36.0 & 37.5 & 87.5 & 20.0 & 30.0 & 20.0 & 66.7 & 22.2 & 40.0 \\
\hline Mild & 0 & 4.2 & 0.0 & 16.0 & 12.5 & 50.0 & 0.0 & 25.0 & 20.0 & 16.7 & 22.2 & 20.0 \\
\hline Moderate & 14.3 & 4.2 & 12.5 & 12.0 & 12.5 & 25.0 & 20.0 & 5.0 & 0.0 & 0.0 & 0.0 & 0.0 \\
\hline Severe & 42.9 & 25 & 0.0 & 8.0 & 12.5 & 12.5 & 0.0 & 0.0 & 0.0 & 50.0 & 0.0 & 20.0 \\
\hline \% vomiting & 42.9 & 33.3 & 0.0 & 8.0 & 0.0 & 25.0 & 40.0 & 15.0 & 0.0 & 0.0 & 0.0 & 0.0 \\
\hline Mild & 0 & 4.2 & 0.0 & 4.0 & 0.0 & 12.5 & 40.0 & 5.0 & 0.0 & 0.0 & 0.0 & 0.0 \\
\hline Moderate & 14.3 & 4.2 & 0.0 & 0.0 & 0.0 & 0.0 & 0.0 & 5.0 & 0.0 & 0.0 & 0.0 & 0.0 \\
\hline Severe & 28.6 & 25 & 0.0 & 4.0 & 0.0 & 12.5 & 0.0 & 5.0 & 0.0 & 0.0 & 0.0 & 0.0 \\
\hline \% lightheaded & 57.1 & 47.8 & 25.0 & 28.0 & 25.0 & 25.0 & 20.0 & 10.0 & 0.0 & 30.0 & 11.1 & 0.0 \\
\hline Mild & 14.3 & 13.0 & 12.5 & 16.0 & 25.0 & 25.0 & 0.0 & 10.0 & 0.0 & 33.3 & 11.1 & 0.0 \\
\hline Moderate & 14.3 & 26.1 & 0.0 & 4.0 & 0.0 & 0.0 & 20.0 & 0.0 & 0.0 & 16.7 & 0.0 & 0.0 \\
\hline Severe & 28.6 & 8.7 & 12.5 & 8.0 & 0.0 & 0.0 & 0.0 & 0.0 & 0.0 & 0.0 & 0.0 & 0.0 \\
\hline$\%$ fever & 42.9 & 25.0 & 0.0 & 4.0 & 12.5 & 25.0 & 0.0 & 0.0 & 0.0 & 0.0 & 0.0 & 0.0 \\
\hline Mild & 28.6 & 16.7 & 0.0 & 4.0 & 12.5 & 25.0 & 0.0 & 0.0 & 0.0 & 0.0 & 0.0 & 0.0 \\
\hline Moderate & 14.3 & 4.2 & 0.0 & 0.0 & 0.0 & 0.0 & 0.0 & 0.0 & 0.0 & 0.0 & 0.0 & 0.0 \\
\hline Severe & 0.0 & 4.2 & 0.0 & 0.0 & 0.0 & 0.0 & 0.0 & 0.0 & 0.0 & 0.0 & 0.0 & 0.0 \\
\hline \% intravenous fluid & 0.0 & 25.0 & 10.0 & 30.7 & 0 & 12.5 & 20.0 & 5.0 & 0.0 & 0.0 & 0.0 & 0.0 \\
\hline $\begin{array}{l}\text { \% early abx. treatment } \\
\% \text { seroconversion }\end{array}$ & 85.7 & 70.8 & 20.0 & 46.2 & 12.5 & 75.0 & 60.0 & 30.0 & 20.0 & 0.0 & 0.0 & 0.0 \\
\hline \multicolumn{13}{|l|}{$\begin{array}{l}\text { \% seroconversion } \\
2 \text {-fold }\end{array}$} \\
\hline LT IgG & 57.1 & 95.8 & 100.0 & 92.3 & 75.0 & 62.5 & 80.0 & 80.0 & 80.0 & 83.3 & 66.7 & 40.0 \\
\hline LT IgA & 71.4 & 91.7 & 100.0 & 69.2 & 87.5 & 62.5 & 80.0 & 65.0 & 60.0 & 50.0 & 55.6 & 40.0 \\
\hline CF IgG & 100.0 & 70.8 & $40.0^{\mathrm{a}}$ & $7.7^{\mathrm{a}}$ & 37.5 & 12.5 & 100.0 & 100.0 & 60.0 & 100.0 & 88.9 & 20.0 \\
\hline $\begin{array}{l}\text { CF IgA } \\
\text { 4-fold }\end{array}$ & 100.0 & 50.0 & $70.0^{\mathrm{a}}$ & $11.5^{\mathrm{a}}$ & 50.0 & 12.5 & 100.0 & 95.0 & 100.0 & 83.3 & 100.0 & 40.0 \\
\hline LT IgG & 42.9 & 83.3 & 100.0 & 88.5 & 37.5 & 25.5 & 80.0 & 75.0 & 60.0 & 50.0 & 44.4 & 20.0 \\
\hline LT IgA & 42.9 & 83.3 & 90.0 & 65.4 & 62.5 & 50.0 & 80.0 & 65.0 & 60.0 & 50.0 & 55.6 & 40.0 \\
\hline CF IgG & 100.0 & 70.8 & 40.0 & 7.7 & 37.5 & 12.5 & 60.0 & 85.0 & 40.0 & 33.3 & 44.4 & 0.0 \\
\hline CF IgA & 100.0 & 50.0 & 40.0 & 3.9 & 50.0 & 12.5 & 100.0 & 85.0 & 60.0 & 83.3 & 100.0 & 40.0 \\
\hline \multicolumn{13}{|l|}{ \% ASC response } \\
\hline LT IgA & 85.7 & 79.2 & 100.0 & 76.9 & 100.0 & 75.0 & ND & ND & ND & ND & ND & ND \\
\hline CF IgA & 100.0 & 37.5 & $100.0^{\mathrm{b}}$ & $96.2^{\mathrm{b}}$ & 87.5 & 12.5 & ND & ND & ND & ND & ND & ND \\
\hline
\end{tabular}

a Shown are response rates to CS3 (CS1 response rates $1 \times 10^{8}: \operatorname{IgG}=50.0, \operatorname{IgA}=70.0 ; 1 \times 10^{9}: \operatorname{IgG}=69.2, \operatorname{Ig} A=65.4$ ).

b Shown are response rates to CS3 (CS1 response rates $1 \times 10^{8}=100.0 ; 1 \times 10^{9}=92.3$ ). 
Table 4

Description of diarrheal episode \{presented as median (interquartile range)\} among subjects meeting diarrhea definition by strain and approximate dose.

\begin{tabular}{|c|c|c|c|c|c|c|c|}
\hline Strain & $\begin{array}{l}\text { Approx. } \\
\text { dose }\end{array}$ & \# Loose stools & Total volume & $\begin{array}{l}\text { Time to first loose } \\
\text { stool }\end{array}$ & $\begin{array}{l}\text { Maximum } 24 \mathrm{~h} \\
\text { volume }\end{array}$ & Duration & $\begin{array}{l}\text { Time to antibiotic } \\
\text { treatment }\end{array}$ \\
\hline \multirow[t]{2}{*}{ H10407 } & $1 \mathrm{E} 8$ & $8.5(5.0,17.0)$ & $1923.0(1125.0,2673.0)$ & $47.8(29.2,62.2)$ & $\begin{array}{l}948.5(573.0, \\
1243.0)\end{array}$ & $81.3(33.0,118.2)$ & $81.6(57.6,103.2)$ \\
\hline & $1 \mathrm{E} 9$ & $10.0(8.0,16.0)$ & $1643.8(1325.0,2379.3)$ & $27.6(24.6,42.2)$ & $\begin{array}{l}984.0(715.5, \\
1534.0)\end{array}$ & $58.4(47.5,84.0)$ & $48.0(38.4,108.2)$ \\
\hline \multirow[t]{2}{*}{ E24377A } & $5 \mathrm{E} 8$ & $6.0(4.0,9.0)$ & $786.5(383.9,1139.0)$ & $11.7(6.6,13.5)$ & $\begin{array}{l}556.1(326.0, \\
845.0)\end{array}$ & $27.3(19.8,29.7)$ & $29.7(17.0,33.2)$ \\
\hline & $5 \mathrm{E} 9$ & $6.0(4.0,8.0)$ & $979.0(479.6,1183.8)$ & $14.2(11.6,22.0)$ & $\begin{array}{l}508.8(386.0, \\
771.0)\end{array}$ & $29.2(10.4,41.3)$ & $119.8(16.5,119.9)$ \\
\hline \multirow[t]{2}{*}{ B7A } & $1 \mathrm{E} 9$ & $5.0(4.0,10.0)$ & $822.0(587.0,915.0)$ & $9.4(7.3,11.0)$ & $\begin{array}{l}435.0(382.0 \\
651.0)\end{array}$ & $42.1(41.9,126.0)$ & $\begin{array}{l}156.0(144.0, \\
156.0)\end{array}$ \\
\hline & $1 \mathrm{E} 10$ & $5.5(3.0,7.5)$ & $825.0(684.5,998.5)$ & $13.9(11.0,15.1)$ & $\begin{array}{l}543.5(491.0 \\
809.5)\end{array}$ & $30.0(13.6,50.8)$ & $60.0(36.0,132.0)$ \\
\hline \multirow[t]{3}{*}{$\begin{array}{l}\text { LSN03- } \\
\text { 016011/A }\end{array}$} & $5 \mathrm{E} 8$ & $7.0(4.0,10.0)$ & $753.3(556.2,1612.5)$ & $50.0(24.9,87.4)$ & $\begin{array}{l}556.2(264.0, \\
1033.5)\end{array}$ & $47.3(25.2,71.3)$ & $120.3(96.3,120.3)$ \\
\hline & $5 \mathrm{E} 9$ & $8.0(5.0,10.0)$ & $910(624.7,1209.6)$ & $22.6(17.7,26.6)$ & $\begin{array}{l}771.2(519.0, \\
999.0)\end{array}$ & $35.6(15.5,54.3)$ & $48.3(24.3,120.3)$ \\
\hline & $5 \mathrm{E} 8$ & $5.0(-)$ & $1186.0(-)$ & $16.7(-)$ & $1186.0(-)$ & $10.0(-)$ & $27.0(-)$ \\
\hline \multirow[t]{2}{*}{ WS0115A } & $5 \mathrm{E} 9$ & $6.0(5.0,7.0)$ & $676.5(402.0,951.0)$ & $6.8(5.1,8.4)$ & $\begin{array}{l}448.5(402.0, \\
495.0)\end{array}$ & $46.9(22.2,71.5)$ & $120.0(-)$ \\
\hline & $1 \mathrm{E} 10$ & $4.5(3.5,5.0)$ & $737.8(420.5,927.0)$ & $25.1(19.6,26.3)$ & $\begin{array}{l}439.8(391.1, \\
624.4)\end{array}$ & $36.0(26.0,53.8)$ & $120.0(-)$ \\
\hline DS26-1 & $5 \mathrm{E} 8$ & - & - & - & - & - & - \\
\hline
\end{tabular}

the low and high doses of $\mathrm{H} 10407$ until the $96 \mathrm{~h}$ mark where they are exceeded by the low dose of H10407. The other dose/strain combinations show increases in stool volume and count through the $72-84 \mathrm{~h}$ post-inoculation timepoint at which point they tend to level off. Importantly, there was variability in the median time to antibiotic treatment (Table 4), although this alone does not account for the temporal variability in diarrhea output.

Using multivariate models, we found several important predictors of clinical outcomes. The risk of diarrhea, malaise and headache was variable by ETEC strain. Specifically, when using H10407 as the reference, subjects receiving the WS0115A strain had a significantly decreased risk of diarrhea (Relative Risk $\{R R\}=0.63, p=0.03$ ) and headache ( $R R=0.47, p=0.04$ ). Similarly, the risk of headache was decreased in subjects receiving either the LSN03-016011/A $(\mathrm{RR}=0.35, p<0.01)$ or $\mathrm{E} 24377 \mathrm{~A}(\mathrm{RR}=0.44, p<0.01)$ strains and subjects receiving the $B 7 A$ strain had a borderline significant decreased risk of malaise ( $R R=0.36, p=0.06)$. Regardless of strain administered, the risk of headache increased with increasing inoculum dose ( $R R=1.23, p=0.05)$ and was higher in females than in males $(R R=1.39, p=0.07)$. Being of female gender also increased the risk of

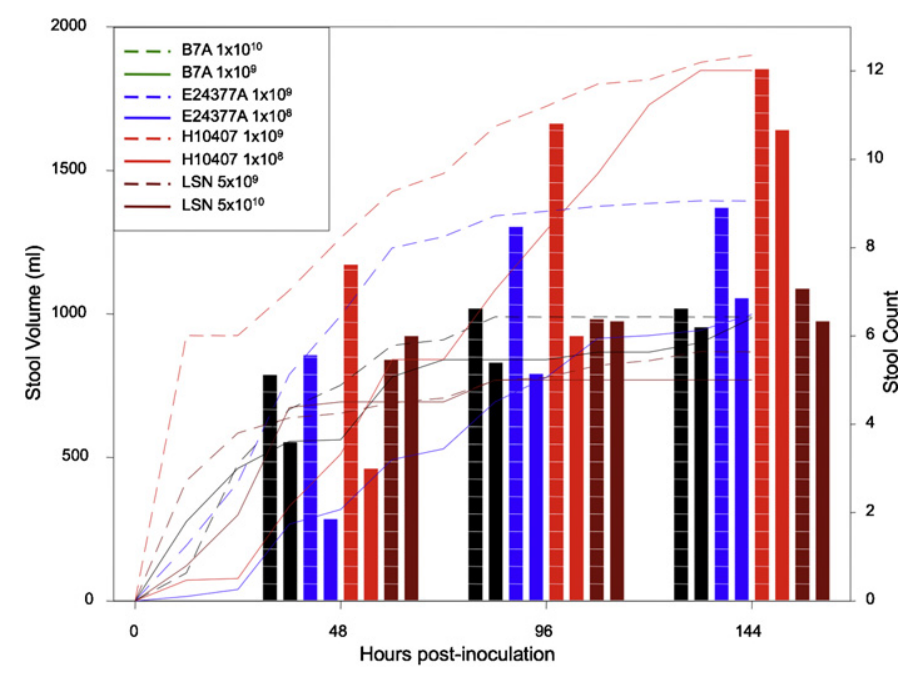

Fig. 3. Volume and number of loose stools over observation period by strain and dose. Bars: stool count; Lines: stool volume. reporting nausea $(\mathrm{RR}=1.92, p<0.01)$ and abdominal pain or cramps $(\mathrm{RR}=1.39, p=0.07)$. The risk of nausea was also higher in subjects of Caucasian race $(R R=1.49, p=0.04)$, compared to those of AfricanAmerican race. We found no effect of dose on the risk of any other outcomes (other than headache) likely due to the narrow range of relatively high inocula evaluated. In contrast, increasing baseline LT IgG levels were associated with a decreased risk of diarrhea $(\mathrm{RR}=0.85, p=0.02)$. However, this association was not consistent when stratified by diarrhea severity (data not shown). There was no association with baseline LT IgA or any titers to the homologous colonization factors.

\subsubsection{Immunology data}

Serologic and ASC responses to LT and homologous colonization factor were relatively common for all dose/strain combinations. Overall, baseline serologic titers to LT (IgA GMT: 85, IgG GMT: 10) were low across all studied dose/strain combinations as were serologic titers to homologous fimbriae (CFA/I: IgA GMT:4, IgG GMT:16; CS1: IgA GMT:296, IgG GMT:764; CS3: IgA GMT:376, IgG GMT:378; CS6: IgA GMT:4, IgG GMT:17; CS17: IgA GMT:3, IgG GMT:13; CS19: IgA GMT: 17, IgG GMT: 31). Neither anti-LT IgA baseline titers nor anti-CF IgG or IgA appeared to correlate with diarrhea risk or severity. Similarly, baseline levels of antibody secreting cells (ASCs) specific to either LT or the homologous CF were low with only 5 subjects presenting with at least 1 ASC per $10^{6}$ peripheral blood mononuclear cells (PBMC). All 5 subjects had diarrhea postinoculation ( 1 severe, 2 moderate and 2 mild) likely reflecting no association between low level ASCs at baseline and diarrhea risk following inoculation.

Early antibiotic treatment did not appear to reduce the frequency or magnitude of serologic or ASC responses to LT or homologous CF with the majority of subjects exhibiting a response to both antigens (data not shown). Additionally, there was no significant association between the time to antibiotic treatment and maximum LT or CF titers (data not shown). Similarly, the duration of the subject's diarrheal episode had no significant impact on resultant maximum LT or CF titers (data not shown).

\section{Discussion}

A systematic review of 27 studies on 11 ETEC strains highlights variability in inoculum preparation and administration as well as 


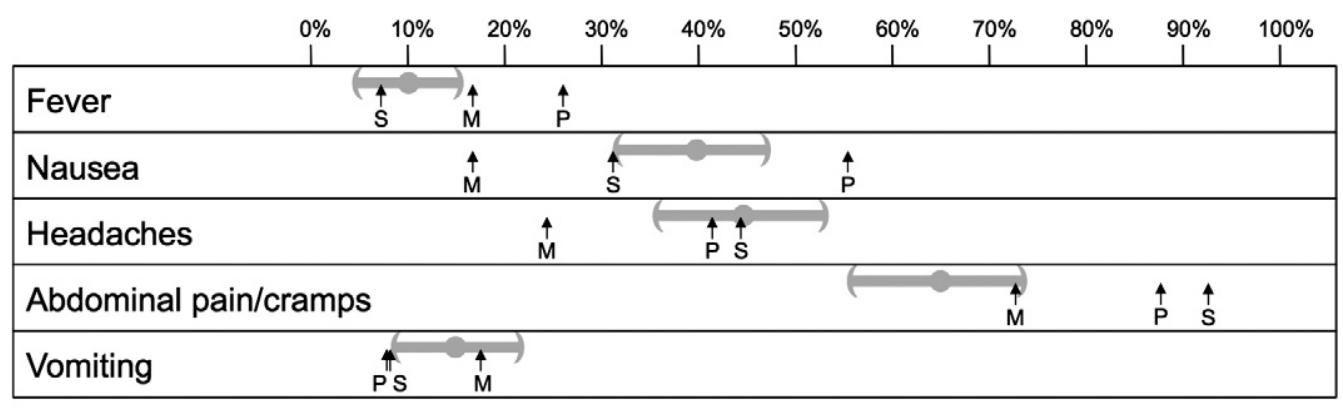

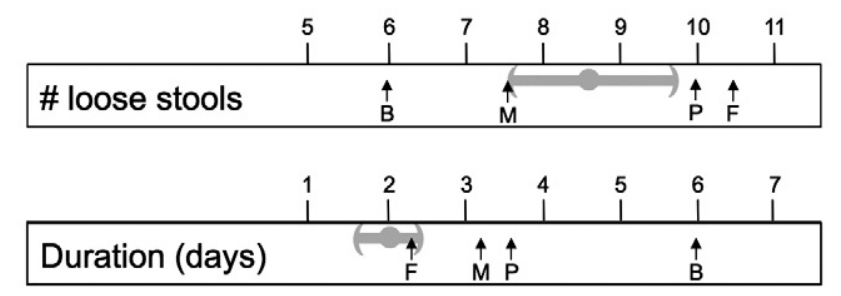

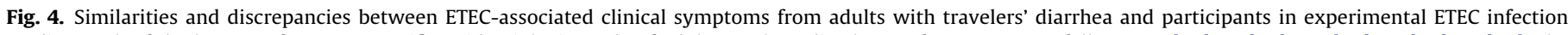

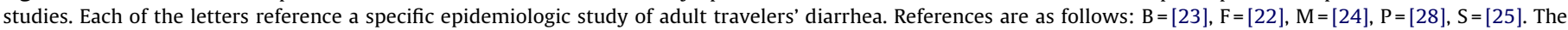
represents the average point estimate with $95 \%$ confidence intervals calculated from the individual patient-level data.

differences in clinical and immunological outcomes across studies. We were able to calculate general estimates of diarrhea attack rates for the 3 most utilized strains, B7A, E24377A and H10407; however, report variability complicated calculations of aggregate outcome measures for given strains and/or doses. Furthermore, utilization of individual patient-level data enabled summative estimates of disease severity parameters and identification of strain and host-specific factors associated with specific clinical outcomes.

\subsection{Report variability}

A total of 27 different studies of ETEC experimental infection have been performed to date, the majority of which are available in the peer-reviewed literature. Of the published studies, variability existed in data reported and methods of analysis. Specifically, while all authors provided information on the number of subjects that met the primary diarrhea outcome, very few provided detailed information on the disease course of those subjects. Additional information on other ETEC-associated symptoms, including their severity, requirements for intravenous therapy, time from antibiotic treatment to microbial cure, and duration of ETEC-associated symptoms after antibiotic treatment would guide future protocol and informed consent development. Additionally, specific summary measures describing the diarrheal episode such as total stool volume, number of stools passed and time to event information would be of equal importance to increase comparability of data across different strains and doses. In an effort to standardize reporting and identify minimum data elements from experimental infection studies, we recommend a consortium be formed to define critical data elements similar to what has been done with travelers' diarrhea treatment trials [20].

\subsection{Similarity to natural infections}

To date, the results of experimental infection models have shown a great diversity in disease severity across a range of doses for a given strain, and among ETEC strains at similar doses. Some have argued that the most rigorously studied strains induce ETEC disease inconsistent with that observed in natural settings such as among naive travelers to an endemic region [21]. While there are numerous epidemiological studies on traveler's diarrhea, few have reported ETEC-specific outcomes and clinical presentation in a naive, adult population. As shown in Fig. 4, the estimates from those studies can be directly compared to estimates obtained from human challenge studies described here.

Most recently, Frech et al. described the results of a placebocontrolled clinical trial evaluating an LT skin patch vaccine in travelers to Guatemala or Mexico [22]. Placebo recipients with ETEC-attributable disease experienced a median of 2.2 days of diarrhea and a median of 10.5 (range: 5-30) loose stools. A slightly longer duration (median of 6 days) and slightly lower total number of loose stools (median of 6 loose stools) was reported by Bolin et al. in a similar population traveling to these 2 countries [23]. Among Finnish travelers to Morocco, Matilla et al. reported a median diarrhea duration of 3.1 days and a total output of 7.5 loose stools with onset occurring approximately 6 days after arrival into the country [24]. In addition to diarrhea, subjects with ETEC also reported abdominal pain (71\%), nausea/vomiting (18\%), fever (18\%), headaches (24\%) and myalgias (19\%). The rates of these concurrent ETEC-related symptoms are similar to those reported by Sanders et al. in U.S. military personnel involved in a training exercise in Thailand [25].

Although in these studies ETEC has tended to be classified as a more mild disease, it can clearly present as a more severe dehydrating illness requiring intravenous rehydration [26]. The drivers of disease severity are largely unknown; though likely represent an interplay between host and pathogen factors. One factor that has inconsistently shown a significant association with increased disease severity is ST production either alone or in combination with LT $[27,28]$. This is consistent with what has been observed in experimental infection studies, although the data for LT-only producing strains are limited. To date, only 4 LT-only strains have been administered: LSN03-016011/A, DS26-1, E2528-C1, TD255-C4 (Table 2). While doses have ranged from 5E8 to 1E10, diarrhea attack rates have been relatively low across the dose/strain combinations with relatively infrequent and less severe associated symptoms. However, this clearly does not explain all the variation observed within the experimental infection studies as strains producing ST only and LT and ST in combination have resulted in a continuum (i.e., from mild to severe) of clinical symptoms at various inoculum levels. 
Table 5

Products assessed using the ETEC human challenge model.

\begin{tabular}{|c|c|c|c|c|c|c|}
\hline Reference & Year & Author & Product (route) & Strain & Dose (cfu) & Result \\
\hline \multirow[t]{5}{*}{ [64] } & 1981 & Clements & $\begin{array}{l}\text { Lactobacillus } \\
\text { acidophilus }\end{array}$ & TD225-C4 & $1 \times 10^{10}$ & No protection against diarrhea \\
\hline & & & $\begin{array}{l}\text { Lactobacillus } \\
\text { bulgaricus (oral) }\end{array}$ & $214-4$ & $1 \times 10^{8}$ & No protection against diarrhea \\
\hline & & & & H10407 & $1 \times 10^{8}$ & No protection against diarrhea \\
\hline & & & & B7A & $1 \times 10^{8}$ & No protection against diarrhea \\
\hline & & & & B7A & $1 \times 10^{10}$ & No protection against diarrhea \\
\hline \multirow[t]{4}{*}{ [65] } & 1982 & Levine & $\begin{array}{l}\text { Type } 1 \text { somatic pili } \\
\text { (intramuscular) }\end{array}$ & H10407 & $5 \times 10^{8}$ & $\begin{array}{l}\text { Significant protection from } \\
\text { diarrhea }\end{array}$ \\
\hline & & & & H10407 & $5 \times 10^{8}$ & $\begin{array}{l}\text { Nonsignificant decrease } \\
\text { (37.5\%) in diarrhea attack rates } \\
\text { compared to controls }\end{array}$ \\
\hline & & & & H10407 & $1 \times 10^{7}$ & No protection against diarrhea \\
\hline & & & & $\mathrm{B} 7 \mathrm{~A}$ & $1 \times 10^{10}$ & No protection against diarrhea \\
\hline$[14]$ & 1983 & Graham & $\begin{array}{l}\text { Bismuth subsalicylate } \\
\text { (oral) }\end{array}$ & H10407 & $1 \times 10^{8}$ & $\begin{array}{l}\text { Significant protection from } \\
\text { diarrhea }\end{array}$ \\
\hline \multirow[t]{3}{*}{ [66] } & 1984 & Evans & CFA/I and CFA/II (oral) & H10407 & $4 \times 10^{8}$ & No protection against diarrhea \\
\hline & & & & H1765 & $4 \times 10^{8}$ & No protection against diarrhea \\
\hline & & & $\begin{array}{l}\text { CFA/I (subcutaneous } \\
\text { prime followed by two } \\
\text { oral doses } 1 \text { week } \\
\text { apart) }\end{array}$ & H10407 & $4 \times 10^{8}$ & $\begin{array}{l}25 \% \text { lower diarrhea attack rate } \\
\text { compared to subjects receiving } \\
\text { oral vaccination only }\end{array}$ \\
\hline$[16,17]$ & 1986 & Levine & $\begin{array}{l}\text { E1392-75-2A: live } \\
\text { attenuated strain } \\
\text { expressing CS1 and CS3 } \\
\text { (oral) }\end{array}$ & E24377A & $5 \times 10^{8}$ & $\begin{array}{l}\text { Significant protection from } \\
\text { diarrhea }\end{array}$ \\
\hline \multirow[t]{3}{*}{ [30] } & 1988 & Evans & $\begin{array}{l}\text { Killed whole cell strain } \\
\text { H10407 (oral) }\end{array}$ & H10407 & $5 \times 10^{9}$ & $\begin{array}{l}\text { Significant protection from } \\
\text { diarrhea }\end{array}$ \\
\hline & & & & $\mathrm{B} 2 \mathrm{C}$ & $3 \times 10^{8}$ & $\begin{array}{l}\text { Diarrhea attack rate of } 25 \% \text { (no } \\
\text { comparable placebo } \\
\text { comparator }\end{array}$ \\
\hline & & & & CFA/I; O63:H- & Not reported & $\begin{array}{l}\text { Diarrhea attack rate of } 25 \% \text { (no } \\
\text { comparable placebo } \\
\text { comparator) }\end{array}$ \\
\hline [31] & 1988 & Evans & $\begin{array}{l}\text { Killed whole cell strain } \\
\text { H10407 (oral) }\end{array}$ & H10407 & $5 \times 10^{9}$ & $\begin{array}{l}\text { Significant protection from } \\
\text { diarrhea }\end{array}$ \\
\hline$[40]$ & 1988 & Tacket & $\begin{array}{l}\text { Bovine milk IgG against } \\
\text { common ETEC O } \\
\text { serogroups (oral) }\end{array}$ & H10407 & $1.2 \times 10^{9}$ & Significant protection \\
\hline [68] & 1994 & Tacket & $\begin{array}{l}\text { CS1 and CS3 } \\
\text { encapsulated in } \\
\text { biodegradable } \\
\text { microspheres } \\
\text { (intestinal tube) }\end{array}$ & E24377A & $3 \times 10^{9}$ & $\begin{array}{l}\text { Nonsignificant decrease } \\
(30.0 \%) \text { in diarrhea attack rates } \\
\text { compared to controls }\end{array}$ \\
\hline [41] & 1998 & Freedman & $\begin{array}{l}\text { Bovine milk IgG against } \\
\text { CFA/I (oral) }\end{array}$ & H10407 & $1 \times 10^{9}$ & $\begin{array}{l}\text { Significant protection from } \\
\text { diarrhea }\end{array}$ \\
\hline [69] & 1999 & Tacket & $\begin{array}{l}\text { Enteric-coated } \\
\text { capsules containing } \\
\text { bovine milk IgG against } \\
\text { CFA/I, CS3 and CS6 }\end{array}$ & E24377A & $1 \times 10^{8}$ & $\begin{array}{l}\text { Significant protection from } \\
\text { diarrhea }\end{array}$ \\
\hline$[21]$ & 2007 & McKenzie & LT (skin patch) & E24377A & $6 \times 10^{8}$ & $\begin{array}{l}\text { No protection against } \\
\text { moderate to severe diarrhea; } \\
\text { amelioration of disease }\end{array}$ \\
\hline [71] & 2008 & McKenzie & $\begin{array}{l}\text { PTL-003: live } \\
\text { attenuated strain } \\
\text { expressing CS1 and CS3 } \\
\text { (oral) }\end{array}$ & E24377A & $3 \times 10^{9}$ & $\begin{array}{l}\text { No protection against } \\
\text { moderate to severe diarrhea }\end{array}$ \\
\hline
\end{tabular}

\subsection{Model utility}

It can be argued that the ETEC volunteer challenge model is an overly rigorous and artificial method for assessing preliminary efficacy of ETEC vaccine candidates. As reported by McKenzie et al., an LT skin patch vaccine failed to protect against diarrhea in an experimental infection study with the strain E34277A, although there were trends toward a less severe illness in the vaccinated subjects [21]. However, when that vaccine (with minor modifications in the administration) was evaluated in a relatively small logistics field trial, a statistically significant reduction was observed in the rate of moderate to severe diarrhea of any cause and in the number of loose stools and duration of illness among those who developed ETECassociated diarrhea [22]. Those data were not corroborated in an expanded field trial and subsequent development of the ETEC patch vaccine was halted [29]. While the results of the expanded efficacy study have not been released in detail, it would be of interest to assess the correlation between efficacy effect estimates between that study and the original vaccination/challenge study.

To date, the only ETEC vaccine that has shown statistically significant protection in vaccination-challenge studies is the oral, colicin E2 treated, whole-cell vaccine evaluated by Evans et al. [30,31]. However, lack of additional clinical development of that vaccine precludes any assessment of the 
utility of the human challenge model to predict field efficacy.

Bismuth subsalicylate has been shown to afford significant protection (protective efficacy $=76.3 \%$ ) against ETEC-associated diarrhea in the experimental infection model $[14,15]$. These results correlated well with several field studies evaluating the efficacy of bismuth subsalicylate against all-cause diarrhea [32]. Specifically, in field studies of diarrhea in travelers to endemic regions, prophylaxis with bismuth subsalicylate yielded an approximate $65 \%$ efficacy against all-cause diarrhea [33-35]. While bismuth subsalicylate prophylaxis is not the same as active vaccination, these data highlight the potential utility of these models in predicting outcomes in large scale clinical trials in travelers to ETEC endemic settings.

Although markedly different than disease prevention, Black et al. assessed trimethoprim and trimethoprim-sulfamathoxazole (Bactrim) in the treatment of subjects with experimentally induced ETEC diarrhea [36]. They saw a significant decrease in the number of loose stools and in diarrhea duration among those receiving the study drugs compared to those receiving placebo. This is consistent with studies of travelers' diarrhea treatment with these antibiotics [37-39].

Tacket et al. evaluated the ability of bovine milk antibodies raised against ETEC expressing CFA/I to confer passive protection against diarrhea in volunteers challenged with H10407 [40]. This was followed by a study by Freedman et al. demonstrating significant protection utilizing bovine milk antibodies against only the CFA/I fimbriae [41]. Unfortunately, despite these positive findings, no field studies utilizing either of these formulations have been conducted precluding a direct comparison between the two settings.

A listing of the prophylactic products assessed using the ETEC human challenge model is included in Table 5, and a review of ETEC vaccine candidates, including those assessed using these models has been previously reported [42]. It should be noted that the studies referenced above only compare and contrast field epidemiological studies with experimental infection studies in adults. This fails to represent the population with the highest disease burden, children living in ETEC endemic regions. This population is not one in which experimental ETEC infection would be ethically justifiable. This represents a potential challenge even if the experimental infection model is deemed an adequate predictor of field efficacy in an adult traveler population.

\subsection{Unique severity with $\mathrm{H} 10407$}

It has long been recognized that ETEC strain H10407 causes significant disease in experimental infection studies. Although other ETEC strains with similar diarrhea attack rates have been evaluated, H10407 appears to induce a more severe diarrhea. For example, in the study by McKenzie et al. evaluating a bovine milk IgG product, four of the eleven placebo recipients (36\%) had over 10 diarrheal stools totaling 2.0-6.5 L. This is similar to the amount of fluid loss seen following an infection with Vibrio cholera 01 [43]. In addition to the significant diarrheal output, H10407 is associated with more severe concurrent signs and symptoms with fever and vomiting reported in a relatively high proportion of subjects compared to volunteers challenged with other ETEC strains. Efforts are ongoing to evaluate lower inoculum doses of H10407 [44]. These studies may identify lower inoculum doses that retain a high diarrhea attack rate yet result in a lower frequency of high volume output and less severe concurrent symptoms.

A number of proposed virulence factors have been identified for H10407 including EAST [45], Tia [46,47], Tib [47], leoA [48],
EatA [49] and EtpA [50]. However, studies to date are unclear as to the effect each may play in the pathogenesis observed in experimental human infections and may not fully explain the differential effect seen with this particular strain compared to other ETEC challenge strains administered to date. The human challenge model could be used to compare the disease severity produced by $\mathrm{H} 10407$ compared to isogenic mutants lacking one or more of these genes. Additionally, this model could be utilized to evaluate host genomics and differences in innate and adaptive immune responses and effects on the host's microbiome.

\subsection{Ethics}

All clinical trials must weigh the ethical dilemmas involved with putting human subjects at risk against the potential benefit of a future drug, vaccine or other product designed to treat or prevent disease. This is no different in experimental ETEC infections, and in fact may be more difficult to appropriately balance the risk-benefit ratio. The ethical framework developed by Miller and Grady offers a structure by which to evaluate experimental infection studies and can serve as a guide for future ETEC infection research [51]. They highlight seven ethical issues that should be used to evaluate proposed studies, the most pertinent of which is "Risk". While ETEC-associated diarrhea in adults has generally been thought of as a relatively mild, self-limited disease, recent literature has highlighted a potential association with post-infectious sequelae. Two separate meta-analyses have shown a 7-fold increase in the risk of developing post-infectious irritable bowel syndrome (PI-IBS), following an episode of acute infectious gastroenteritis (IGE), of which ETEC is a common cause [52,53] and two studies have found this association in areas of high ETEC prevalence [54,55]. Although the pathogen-specific attributable risk for this post-infectious sequelae is currently unknown, these data suggest a new paradigm in the understanding of infectious diarrhea, including ETEC, and its impact on long-term health. In addition, a growing body of literature has shown a similar association between IGE and inflammatory bowel disease (IBD) [56-58]. Although unlikely to be identified in post-infection follow-ups due to its generally rare occurrence, this association highlights the need for further study of a pathogen that was once thought to cause only a self-limiting diarrhea.

Based on the relatively high inoculum required to elicit diarrhea in a sufficient number of subjects and the inverse association between diarrhea risk and baseline LT IgG titers, one may hypothesize that inclusion of serologic screening, as has been utilized for Campylobacter jejuni challenge model development [59,60], may decrease the required inoculum. None of the studies reviewed here reported serologic screening of potential study participants. However, several did report an exclusion for subjects with prior travel to ETEC-endemic regions and/or exclusion for known ETEC exposure. It is unclear what the added impact of baseline serologic screening may have on future utilization of the model.

The experimental human ETEC challenge has proved invaluable in defining and understanding strain pathogenicity, highlighting strain variability in disease manifestation and increasing our knowledge of the human immune response. It has also served as an important tool in the evaluation of vaccines and other experimental therapies. The information gleaned from these studies should guide future experimental ETEC challenge studies of existing and novel ETEC strains. With our expanding understanding of genomics, proteomics and microbiomics, data obtained from human challenge models may provide the opportunity to gain a better understanding of pathogenesis, host factors impacting susceptibility as well as develop more, well-characterized and targeted vaccines and adjuvants thereby increasing efficacy. 


\section{Copyright statement}

Some authors are employees of the U.S. Government or military service members. This work was prepared as part of official duties. Title 17 U.S.C. $§ 105$ provides that 'Copyright protection under this title is not available for any work of the United States Government.' Title 17 U.S.C. $§ 101$ defines a U.S. Government work as a work prepared by a military service member or employee of the U.S. Government as part of that person's official duties.

\section{References}

[1] DuPont HL, Formal SB, Hornick RB, Snyder MJ, Libonati JP, Sheahan DG, et al. Pathogenesis of Escherichia coli diarrhea. N Engl J Med 1971;285(1):1-9.

[2] Bill and Melinda Gates Foundation. http://www.gatesfoundation.org; accessed 26 February 2010.

[3] Svennerholm AM, Tobias J. Vaccines against enterotoxigenic Escherichia coli. Expert Rev Vaccines 2008;7(6):795-804.

[4] Cryz Jr SJ, Kaper J, Tacket C, Nataro J, Levine MM. Vibrio cholerae CVD103-HgR live oral attenuated vaccine: construction, safety, immunogenicity, excretion and non-target effects. Dev Biol Stand 1995;84:237-44

[5] Moorthy VS, Diggs C, Ferro S, Good MF, Herrera S, Hill AV, et al. Report of a consultation on the optimization of clinical challenge trials for evaluation of candidate blood stage malaria vaccines, 18-19 March 2009, Bethesda, MD, USA. Vaccine 2009;27(42):5719-25.

[6] Egger M, Smith GD, Altman DG. Systematic reviews in health care. 2nd ed. London, GB: BMJ Publishing Group; 2001. p. 487.

[7] Shojania KG, et al. Chapter 3. Evidence-based review methodology. In: Making health care safer: a critical analysis of patient safety practices. University of California at San Francisco (UCSF)-Stanford University Evidence-based Practice Center; 2001. p. 29-38.

[8] Simmonds MC, Higgins JP, Stewart LA, Tierney JF, Clarke MJ, Thompson SG. Meta-analysis of individual patient data from randomized trials: a review of methods used in practice. Clin Trials 2005;2(3):209-17.

[9] DerSimonian R, Laird N. Meta-analysis in clinical trials. Control Clin Trials 1986;7(3):177-88.

[10] Higgins JP, Whitehead A, Turner RM, Omar RZ, Thompson SG. Metaanalysis of continuous outcome data from individual patients. Stat Med 2001;20(15):2219-41.

[11] Turner RM, Omar RZ, Yang M, Goldstein H, Thompson SG. A multilevel model framework for meta-analysis of clinical trials with binary outcomes. Stat Med 2000;19(24):3417-32.

[12] Evans DG, Satterwhite TK, Evans Jr DJ, DuPont HL. Differences in serological responses and excretion patterns of volunteers challenged with enterotoxigenic Escherichia coli with and without the colonization factor antigen. Infect Immun 1978;19(3):883-8.

[13] Satterwhite TK, Evans DG, DuPont HL, Evans Jr DJ. Role of Escherichia coli colonisation factor antigen in acute diarrhoea. Lancet 1978;2(8082):181-4.

[14] Graham DY, Estes MK, Gentry LO. Double-blind comparison of bismuth subsalicylate and placebo in the prevention and treatment of enterotoxigenic Escherichia coli-induced diarrhea in volunteers. Gastroenterology 1983;85(5):1017-22.

[15] Graham DY, Evans DG. Prevention of diarrhea caused by enterotoxigenic Escherichia coli: lessons learned with volunteers. Rev Infect Dis 1990;12(Suppl. 1):S68-72.

[16] Levine M, Morris JG, Losonsky G, Boedeker EC, Rowe B. Fimbriae (pili) adhesins as vaccines. In: Lark DL, Mormakr S, Uhlin B-E, Wolf-Wath H, editors. Protein-carbohydrate interactions in biologic systems: the molecular biology of microbial pathogenicity. London: Academic Press; 1986. p. 143-5.

[17] Tacket CO, Levine MM. Vaccines against enterotoxigenic Escherichia coli infections: part ii: live oral vaccines and subunit (purified fimbriae and toxin subunit) vaccines. In: Levine MM, Woodrow GC, Kaper JB, Cobon GS, editors. New generation vaccines. second ed. New York: Marcel Dekker, Inc.; 1997. p. 879.

[18] Lanata CF, Kaper JB, Baldini MM, Black RE, Levine MM. Sensitivity and specificity of DNA probes with the stool blot technique for detection of Escherichia coli enterotoxins. J Infect Dis 1985;152(5):1087-90.

[19] Levine MM, Bergquist EJ, Nalin DR, Waterman DH, Hornick RB, Young CR, et al. Escherichia coli strains that cause diarrhoea but do not produce heat-labile or heat-stable enterotoxins and are non-invasive. Lancet 1978;1(8074):1119-22.

[20] DuPont HL, Cooperstock M, Corrado ML, Fekety R, Murray DM. Evaluation of new anti-infective drugs for the treatment of acute infectious diarrhea. Infectious Diseases Society of America and the Food and Drug Administration. Clin Infect Dis 1992;15(Suppl 1):S228-35.

[21] McKenzie R, Bourgeois AL, Frech SA, Flyer DC, Bloom A, Kazempour K, et al. Transcutaneous immunization with the heat-labile toxin (LT) of enterotoxigenic Escherichia coli (ETEC): protective efficacy in a double-blind, placebo-controlled challenge study. Vaccine 2007;25(18):3684-91.

[22] Frech SA, Dupont HL, Bourgeois AL, McKenzie R, Belkind-Gerson J, Figueroa JF, et al. Use of a patch containing heat-labile toxin from Escherichia coli against travellers' diarrhoea: a phase II, randomised, double-blind, placebo-controlled field trial. Lancet 2008;371(9629):2019-25.
[23] Bolin I, Wiklund G, Qadri F, Torres O, Bourgeois AL, Savarino S, Svennerholm AM. Enterotoxigenic Escherichia coli with STh and STp genotypes is associated with diarrhea both in children in areas of endemicity and in travelers. J Clin Microbiol 2006;44(11):3872-7.

[24] Mattila L. Clinical features and duration of traveler's diarrhea in relation to its etiology. Clin Infect Dis 1994;19(4):728-34.

[25] Sanders JW, Isenbarger DW, Walz SE, Pang LW, Scott DA, Tamminga C, et al. An observational clinic-based study of diarrheal illness in deployed United States military personnel in Thailand: presentsion and outcome of Campylobacter infection. Am J Trop Med Hyg 2002;67(5):533-8.

[26] Qadri F, Svennerholm AM, Faruque AS, Sack RB. Enterotoxigenic Escherichia col in developing countries: epidemiology, microbiology, clinical features, treatment, and prevention. Clin Microbiol Rev 2005;18(3):465-83.

[27] Qadri F, Das SK, Faruque AS, Fuchs GJ, Albert MJ, Sack RB, et al. Prevalence of toxin types and colonization factors in enterotoxigenic Escherichia coli isolated during a 2-year period from diarrheal patients in Bangladesh. J Clin Microbiol 2000;38(1):27-31.

[28] Porter CK, Riddle MS, Tribble DR, Putnam SD, Rockabrand DM, Frenck RW, et al. The epidemiology of travelers' diarrhea in Incirlik, Turkey: a region with a predominance of heat-stabile toxin producing enterotoxigenic Escherichia coli. Diagn Microbiol Infect Dis 2010;66(3):241-7.

[29] Intercell provides update on clinical trials for the patch-based Travelers Diarrhea vaccine. http://www.intercell.com; accessed 15 December 2010, Editor.

[30] Evans DG, Evans Jr DJ, Opekun AR, Graham DY. Non-replicating oral whole cell vaccine protective against enterotoxigenic Escherichia coli (ETEC) diarrhea: stimulation of anti-CFA (CFA/I) and anti-enterotoxin (anti-LT) intestinal IgA and protection against challenge with ETEC belonging to heterologous serotypes. FEMS Microbiol Immunol 1988;1(3):117-25.

[31] Evans Jr DJ, Evans DG, Opekun AR, Graham DY. Immunoprotective oral whole cell vaccine for enterotoxigenic Escherichia coli diarrhea prepared by in situ destruction of chromosomal and plasmid DNA with colicin E2. FEMS Microbiol Immunol 1988;1(1):9-18.

[32] Rao G, Aliwalas MG, Slaymaker E, Brown B. Bismuth revisited: an effective way to prevent travelers' diarrhea. J Travel Med 2004;11(4):239-41.

[33] DuPont HL, Ericsson CD, Johnson PC, de la Cabada FJ. Use of bismuth subsalicylate for the prevention of travelers' diarrhea. Rev Infect Dis 1990;12(Suppl. 1):S64-7.

[34] DuPont HL, Ericsson CD, Johnson PC, Bitsura JA, DuPont MW, de la Cabada FJ. Prevention of travelers' diarrhea by the tablet formulation of bismuth subsalicylate. JAMA 1987;257(10):1347-50.

[35] Steffen R, DuPont HL, Heusser R, Helminger A, Witassek F, Manhart MD, et al. Prevention of traveler's diarrhea by the tablet form of bismuth subsalicylate. Antimicrob Agents Chemother 1986;29(4):625-7.

[36] Black RE, Levine MM, Clements ML, Cisneros L, Daya V. Treatment of experimentally induced enterotoxigenic Escherichia coli diarrhea with trimethoprim, trimethoprim-sulfamethoxazole, or placebo. Rev Infect Dis 1982;4(2):540-5.

[37] Dupont HL, Ericsson CD, Galindo E, Dupont MW, Mendiola Gomez J Antimicrobial therapy of travellers' diarrhoea. Scand J Gastroenterol Suppl 1983;84:99-105.

[38] Thornton SA, Wignall SF, Kilpatrick ME, Bourgeois AL, Gardiner C, Batchelor RA et al. Norfloxacin compared to trimethoprim/sulfamethoxazole for the treatment of travelers' diarrhea among U.S. military personnel deployed to South America and West Africa. Mil Med 1992;157(2):55-8.

[39] Ericsson CD, Johnson PC, Dupont HL, Morgan DR, Bitsura JA, de la Cabada FJ. Ciprofloxacin or trimethoprim-sulfamethoxazole as initial therapy for travelers' diarrhea. A placebo-controlled, randomized trial. Ann Intern Med 1987;106(2):216-20.

[40] Tacket CO, Losonsky G, Link H, Hoang Y, Guesry P, Hilpert H, et al. Protection by milk immunoglobulin concentrate against oral challenge with enterotoxigenic Escherichia coli. N Engl J Med 1988;318(19):1240-3.

[41] Freedman DJ, Tacket CO, Delehanty A, Maneval DR, Nataro J, Crabb JH. Milk immunoglobulin with specific activity against purified colonization factor antigens can protect against oral challenge with enterotoxigenic Escherichia coli. J Infect Dis 1998;177(3):662-7.

[42] Walker RI, Steele D, Aguado T. Analysis of strategies to successfully vaccinate infants in developing countries against enterotoxigenic E. coli (ETEC) disease. Vaccine 2007;25(14):2545-66.

[43] Dhar U, Bennish ML, Khan WA, Seas C, Huq Khan E, Albert MJ, et al. Clinica features, antimicrobial susceptibility and toxin production in Vibrio cholerae 0139 infection: comparison with V. cholerae 01 infection. Trans R Soc Trop Med Hyg 1996;90(4):402-5.

[44] Harro C, DeNearing B, Marcum A, Feller A, Chakraborty S, Hnizda B, et al Development of a low-dose challenge model for evaluation of vaccines for enterotoxigenic E. coli (ETEC) in volunteers. In: 5th International Vaccines for Enteric Diseases Conference. 2009.

[45] Yamamoto T, Echeverria P. Detection of the enteroaggregative Escherichia col heat-stable enterotoxin 1 gene sequences in enterotoxigenic E. coli strains pathogenic for humans. Infect Immun 1996;64(4):1441-5.

[46] Fleckenstein JM, Kopecko DJ, Warren RL, Elsinghorst EA. Molecular characterization of the tia invasion locus from enterotoxigenic Escherichia coli. Infect Immun 1996;64(6):2256-65.

[47] Lindenthal C, Elsinghorst EA. Enterotoxigenic Escherichia coli TibA glycoprotein adheres to human intestine epithelial cells. Infect Immun 2001;69(1):52-7.

[48] Fleckenstein JM, Lindler LE, Elsinghorst EA, Dale JB. Identification of a gene within a pathogenicity island of enterotoxigenic Escherichia coli H10407 
required for maximal secretion of the heat-labile enterotoxin. Infect Immun 2000;68(5):2766-74.

[49] Patel SK, Dotson J, Allen KP, Fleckenstein JM. Identification and molecular characterization of EatA, an autotransporter protein of enterotoxigenic Escherichia coli. Infect Immun 2004;72(3):1786-94.

[50] Roy K, Hamilton D, Allen KP, Randolph MP, Fleckenstein JM. The EtpA exoprotein of enterotoxigenic Escherichia coli promotes intestinal colonization and is a protective antigen in an experimental model of murine infection. Infect Immun 2008;76(5):2106-12.

[51] Miller FG, Grady C. The ethical challenge of infection-inducing challenge experiments. Clin Infect Dis 2001;33(7):1028-33.

[52] Halvorson HA, Schlett CD, Riddle MS. Postinfectious irritable bowel syndrome-a meta-analysis. Am J Gastroenterol 2006;101(8):1894-9, quiz 1942.

[53] Thabane M, Kottachchi DT, Marshall JK. Systematic review and meta-analysis: the incidence and prognosis of post-infectious irritable bowel syndrome. Aliment Pharmacol Ther 2007;26(4):535-44.

[54] Okhuysen PC, Jiang ZD, Carlin L, Forbes C, DuPont HL. Post-diarrhea chronic intestinal symptoms and irritable bowel syndrome in North American travelers to Mexico. Am J Gastroenterol 2004;99(9):1774-8.

[55] Tuteja AK, Talley NJ, Gelman SS, Alder SC, Thompson C, Tolman K, et al. Development of functional diarrhea, constipation, irritable bowel syndrome, and dyspepsia during and after traveling outside the USA. Dig Dis Sci 2008;53(1):271-6.

[56] Garcia Rodriguez LA, Ruigomez A, Panes J. Acute gastroenteritis is followed by an increased risk of inflammatory bowel disease. Gastroenterology 2006;130(6):1588-94.

[57] Porter CK, Tribble DR, Aliaga PA, Halvorson HA, Riddle MS. Infectious gastroenteritis and risk of developing inflammatory Bowel disease. Gastroenterology 2008.

[58] Ternhag A, Torner A, Svensson A, Ekdahl K, Giesecke J. Short- and long-term effects of bacterial gastrointestinal infections. Emerg Infect Dis 2008; $14(1): 143-8$.

[59] Tribble DR, Baqar S, Carmolli MP, Porter C, Pierce KK, Sadigh K, et al. Campylobacter jejuni strain CG8421: a refined model for the study of Campylobacteriosis and evaluation of Campylobacter vaccines in human subjects. Clin Infect Dis 2009;49(10):1512-9.

[60] Tribble DR, Baqar S, Scott DA, Oplinger ML, Trespalacios F, Rollins D, et al. Assessment of the duration of protection in Campylobacter jejuni experimental infection in humans. Infect Immun 2010;78(4):1750-9.

[61] Levine MM, Caplan ES, Waterman D, Cash RA, Hornick RB, Snyder MJ. Diarrhea caused by Escherichia coli that produce only heat-stable enterotoxin. Infect Immun 1977;17(1):78-82.

[62] Levine MM, Nalin DR, Hoover DL, Bergquist EJ, Hornick RB, Young CR. Immunity to enterotoxigenic Escherichia coli. Infect Immun 1979;23(3):729-36.

[63] Levine MM, Rennels MB, Cisneros L, Hughes TP, Nalin DR, Young CR. Lack of person-to-person transmission of enterotoxigenic Escherichia coli despite close contact. Am J Epidemiol 1980;111(3):347-55.
[64] Clements ML, Levine MM, Black RE, Robins-Browne RM, Cisneros LA, Drusano GL, et al. Lactobacillus prophylaxis for diarrhea due to enterotoxigenic Escherichia coli. Antimicrob Agents Chemother 1981;20(1):104-8.

[65] Levine MM, Black RE, Brinton Jr CC, Clements ML, Fusco P, Hughes TP, et al. Reactogenicity, immunogenicity and efficacy studies of Escherichia coli type 1 somatic pili parenteral vaccine in man. Scand J Infect Dis Suppl 1982;33:83-95.

[66] Evans DG, Graham DY, Evans Jr DJ. Administration of purified colonization factor antigens (CFA/I, CFA/II) of enterotoxigenic Escherichia coli to volunteers. Response to challenge with virulent enterotoxigenic Escherichia coli. Gastroenterology 1984;87(4):934-40.

[67] Levine MM, Ristaino P. Marley G, Smyth C, Knutton S, Boedeker E, et al Coli surface antigens 1 and 3 of colonization factor antigen II-positive enterotoxigenic Escherichia coli: morphology, purification, and immune responses in humans. Infect Immun 1984;44(2):409-20.

[68] Tacket CO, Reid RH, Boedeker EC, Losonsky G, Nataro JP, Bhagat H, et al. Entera immunization and challenge of volunteers given enterotoxigenic E. coli CFA/I encapsulated in biodegradable microspheres. Vaccine 1994;12(14):1270-4.

[69] Tacket CO, Losonsky G, Livio S, Edelman R, Crabb J, Freedman D. Lack of prophylactic efficacy of an enteric-coated bovine hyperimmune milk product against enterotoxigenic Escherichia coli challenge administered during a standard meal. J Infect Dis 1999;180(6):2056-9.

[70] Coster TS, Wolf MK, Hall ER, Cassels FJ, Taylor DN, Liu CT, et al. Immune response, ciprofloxacin activity, and gender differences after human experimental challenge by two strains of enterotoxigenic Escherichia coli. Infect Immun 2007;75(1):252-9.

[71] McKenzie R, Darsley M, Thomas N, Randall R, Carpenter C, Forbes E, et al. A double-blind, placebo-controlled trial to evaluate the efficacy of PTL-003, an attenuated enterotoxigenic E. coli (ETEC) vaccine strain, in protecting against challenge with virulent ETEC. Vaccine 2008;26(36):4731-9.

[72] McKenzie R, Porter C, Cantrell J, DeNearing B, O’Dowd A, Grahek S, et al. Volunteer Challenge with Enterotoxigenic Escherichia coli that Express Intestinal Colonization Factor Fimbriae CS17 and CS19. J Infect Dis. doi:10.1093/infdis/JIR220.

[73] Sack DA, Merson MH, Wells JG, Sack RB, Morris GK. Diarrhoea associated with heat-stable enterotoxin-producing strains of Escherichia coli. Lancet 1975;2(7928):239-41.

[74] Scotland SM, McConnell MM, Willshaw GA, Rowe B, Field AM. Properties of wild-type strains of enterotoxigenic Escherichia coli which produce colonization factor antigen II, and belong to serogroups other than 06. J Gen Microbiol $1985 ; 131(9): 2327-33$

[75] Center for Disease Control. Diarrheal illness on a cruise ship caused by enterotoxigenic Escherichia coli. MMWR 1976;25:229.

[76] Evans Jr DJ, Evans DG. Three characteristics associated with enterotoxigenic Escherichia coli isolated from man. Infect Immun 1973;8(3):322-8.

[77] Khalil SB, Cassels FJ, Shaheen HI, Pannell LK, El-Ghorab N, Kamal K, et al. Characterization of an enterotoxigenic Escherichia coli strain from Africa expressing a putative colonization factor. Infect Immun 1999;67(8):4019-26. 